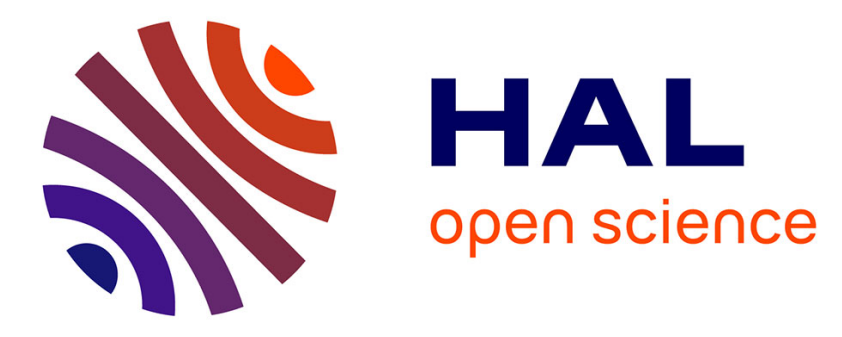

\title{
Field Measurements of Terrestrial and Martian Dust Devils
}

\author{
Jim Murphy, Kathryn Steakley, Matt Balme, Grégoire Déprez, Francesca
}

Esposito, Henrik Kahanpää, Mark Lemmon, Ralph Lorenz, Naomi Murdoch,

Lynn Neakrase, et al.

\section{To cite this version:}

Jim Murphy, Kathryn Steakley, Matt Balme, Grégoire Déprez, Francesca Esposito, et al.. Field Measurements of Terrestrial and Martian Dust Devils. Space Science Reviews, 2016, 203 (1), pp.3987. 10.1007/s11214-016-0283-y . insu-01391498

\section{HAL Id: insu-01391498 \\ https://hal-insu.archives-ouvertes.fr/insu-01391498}

Submitted on 4 Mar 2021

HAL is a multi-disciplinary open access archive for the deposit and dissemination of scientific research documents, whether they are published or not. The documents may come from teaching and research institutions in France or abroad, or from public or private research centers.
L'archive ouverte pluridisciplinaire HAL, est destinée au dépôt et à la diffusion de documents scientifiques de niveau recherche, publiés ou non, émanant des établissements d'enseignement et de recherche français ou étrangers, des laboratoires publics ou privés. 


\section{Open Archive TOULOUSE Archive Ouverte (OATAO)}

OATAO is an open access repository that collects the work of Toulouse researchers and makes it freely available over the web where possible.

This is an author-deposited version published in: http://oatao.univ-toulouse.fr/ Eprints ID: 17289

To cite this version: Murphy, Jim and Steakley, Kathryn and Balme, Matt and Deprez, Gregoire and Esposito, Francesca and Kahanpaa, Henrik and Lemmon, Mark and Lorenz, Ralph and Murdoch, Naomi and Neakrase, Lynn and Patel, Manish and Whelley, Patrick Field Measurements of Terrestrial and Martian Dust Devils. (2016) Space Science Reviews, vol. 203 ( $\mathrm{n}^{\circ}$ 1). pp. 39-87. ISSN 0038-6308

Official URL: http://dx.doi.org/10.1007/s11214-016-0283-y

Any correspondence concerning this service should be sent to the repository administrator: staff-oatao@ listes-diff.inp-toulouse.fr 


\title{
Field Measurements of Terrestrial and Martian Dust Devils
}

\author{
Jim Murphy ${ }^{1}$ (D) Kathryn Steakley ${ }^{1} \cdot$ Matt Balme $^{2} \cdot$ Gregoire Deprez $^{3}$. \\ Francesca Esposito ${ }^{4} \cdot$ Henrik Kahanpää ${ }^{5,6}$ • Mark Lemmon ${ }^{7} \cdot$ Ralph Lorenz $^{8}$.

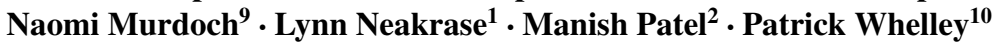

\begin{abstract}
Surface-based measurements of terrestrial and martian dust devils/convective vortices provided from mobile and stationary platforms are discussed. Imaging of terrestrial dust devils has quantified their rotational and vertical wind speeds, translation speeds, dimensions, dust load, and frequency of occurrence. Imaging of martian dust devils has provided translation speeds and constraints on dimensions, but only limited constraints on vertical motion within a vortex. The longer mission durations on Mars afforded by long operating robotic landers and rovers have provided statistical quantification of vortex occurrence (time-of-sol, and recently seasonal) that has until recently not been a primary outcome of more temporally limited terrestrial dust devil measurement campaigns. Terrestrial measurement campaigns have included a more extensive range of measured vortex parameters (pressure, wind, morphology, etc.) than have martian opportunities, with electric field and direct measure of dust abundance not yet obtained on Mars. No martian robotic mission has yet provided contemporaneous high frequency wind and pressure measurements. Comparison of measured terrestrial and martian dust devil characteristics suggests that martian dust devils are larger and possess faster maximum rotational wind speeds, that the absolute magnitude of the pressure deficit within a terrestrial dust devil is an order of magnitude greater
\end{abstract}

\footnotetext{
J. Murphy

murphy@nmsu.edu

1 New Mexico State University, Las Cruces, NM, USA

2 Open University, Milton Keynes, UK

3 Laboratoire Atmosphères, Guyancourt, France

4 INAF, Osservatorio Astronomico di Capodimonte, Naples, Italy

5 Finnish Meteorological Institute, Helsinki, Finland

6 Aalto University/School of Electrical Engineering, Espoo, Finland

7 Texas A\&M University, College Station, TX, USA

8 Johns Hopkins University Applied Physics Lab, Laurel, MD, USA

9 ISAE-SUPAERO, Toulouse University, Toulouse, France

10 NASA Goddard Space Flight Center, Greenbelt, MD, USA
} 
than a martian dust devil, and that the time-of-day variation in vortex frequency is similar. Recent terrestrial investigations have demonstrated the presence of diagnostic dust devil signals within seismic and infrasound measurements; an upcoming Mars robotic mission will obtain similar measurement types.

Keywords Dust devils

\section{Introduction}

Dust devils are small diameter, surface bordering, vertically aligned atmospheric convective vortices made visible by their entrainment of dust lifted from the surface (Fig. 1). Historic anecdotal reports (Lorenz et al. 2016) indicate qualitative awareness of these phenomena extending for millennia into the past. Quantitative awareness is more recent (Ives 1947; Wyett 1954). Dust devils are one mechanism that emplaces dust into atmospheric suspension, affecting air quality, atmospheric clarity, possible hazardous conditions to low flying aircraft, etc. Dust devils have also been identified on Mars, where they play a possibly substantial role in maintaining that planet's persistent atmospheric dust load, especially during the orbital aphelion season (Kahre et al. 2006). It is only during the modern era of scientific investigation and measurement that the true physical understanding of these phenomena on both planets have been achieved. In this presentation we focus upon the quantitative characterization of dust devils, on both Earth and Mars, which have resulted in our current understanding of their physical attributes.

Terrestrial dust devil attributes measured to date include winds (radial, azimuthal, vertical), translation speed, shape/height/width (visualized by suspended dust), central pressure deficit, dust load and its radial and vertical structure and particle size distribution, electric field, and surface dust lifting rate. Measurements have been provided in situ from mobile and stationary instrumented platforms (either individually or in a network) and/or remotely imaged at visible wavelengths, with some infrared wavelength measurements also available. Measurements obtained from Mars' surface include both in situ (pressure, wind) and remotely imaged (visible imaging) characterization, but remain deficient in other areas (electric field, dust lifting rate). While terrestrial field campaigns have usually been, until recently, of short time extent (days, weeks), martian 'campaigns' (robotic exploration missions) have been more time extended, limited by the lifetime of the mission or its most applicable instruments. However, no martian mission has provided continuous sampling for all forms of observations, though the Mars Phoenix Lander did provide almost continuous $0.5 \mathrm{~Hz}$ sampling of its meteorology measurements.

Balme and Greeley (2006) provided an extensive dust devil review. Here we emphasize subsequent gained knowledge in addition to reiterating their primary foci.

In Sect. 2 below we describe primarily surface based imaging characterization of terrestrial dust devils (Sect. 2.1) from a variety of field campaigns, followed by surface based imaging characterization of martian dust devils (Sect. 2.2) provided by the seven spacecraft that have operated/are operating there. [Note that Fenton et al. (2016) in this collection of papers addresses dust devil remote sensing from non-surface based measurements such as those provided by orbiting spacecraft.] This is followed in Sect. 3 by presentation of in situ measurements of terrestrial dust devils (Sect. 3.1) and martian dust devils (Sect. 3.2). 
Fig. 1 Image of a terrestrial dust devil in Eldorado Valley, Nevada, USA, 2009. Notice, for scale, the 'chase vehicle' positioned at the lower left of the dust devil. Image provided by $\mathrm{M}$. Balme

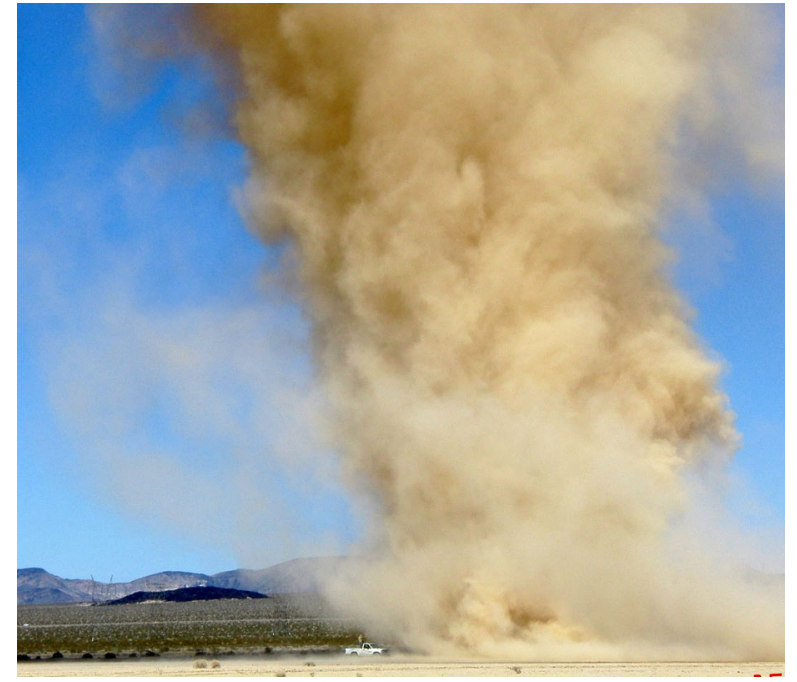

Emphasis is placed upon the types of measurements obtained and their indication of mean characteristics and identified extrema. [Assessment of dust devil population statistics is provided in the accompanying paper by Lorenz and Jackson (2016).] The terrestrial measurements span a large quantity of literature covering a large number of measurements opportunities. The martian literature is more limited and the measurement opportunities much more discrete than their terrestrial counterparts. The martian instrumentation is discussed in some detail. In Sect. 4 we provide a brief discussion of terrestrial versus martian dust devils. In Sect. 5 we address the topic of future measurements desired for a more complete characterization of terrestrial and martian dust devils. Concluding remarks are presented in Sect. 6.

\section{Surface Obtained Imaging of Dust Devils}

Most studies of dust devils on Earth have used either (i) an in-situ sampling methodology (i.e. by 'chasing' a dust devil with an instrumented vehicle, or by waiting for a dust devil to approach one or more immobile instrument stations), or (ii) a local remote sensing methodology, in which one or more observers document the local time, size, dust load, morphology, etc. of dust devils as they occur within some kind of fixed study area. Some studies have used a combination of these approaches by deploying both observers and in-situ instruments.

The main challenge for any field study is that dust devils are inherently changeable and somewhat unpredictable. Hence, measurements of size, lifetime and dustiness of a given dust devil are difficult to make-some dust devils start small and grow larger and dustier and persist for many minutes, whereas similar starting examples can disappear without a trace after just a few seconds. This presents a challenge for any qualitative study as "summarising" any dust devil with a few simple parameters is sometimes impossible. Another problem is that dust devils can travel quickly, and can move into and out of a defined study area (or even the entire field of view of an observer) during their lifetime. Similarly, studies 
Table 1 Terrestrial dust devil observed characteristics

\begin{tabular}{|c|c|c|}
\hline Parameter & Best observations or measurements & Key literature \\
\hline Size (diameter) & $\begin{array}{l}1 \text { to }>100 \mathrm{~m} \text {; Strongly skewed size } \\
\text { frequency distribution: small dust devils } \\
\text { much more common than large; examples } \\
>300 \mathrm{~m} \text { are very uncommon }\end{array}$ & $\begin{array}{l}\text { Carroll and Ryan (1970); Sinclair } \\
\text { (1965); Lorenz (2011) }\end{array}$ \\
\hline Size (height) & $\begin{array}{l}5 \mathrm{~m} \text { to }>1000 \mathrm{~m} \text {; only about } 10 \% \text { are } \\
>300 \mathrm{~m}\end{array}$ & $\begin{array}{l}\text { Sinclair (1965); Bell (1967); } \\
\text { Flower (1936) }\end{array}$ \\
\hline Lifetime & $\begin{array}{l}\text { Seconds to minutes; larger dust devils have } \\
\text { longer lifetimes; some reports of large dust } \\
\text { devils lasting several hours }\end{array}$ & $\begin{array}{l}\text { Flower (1936); Snow and } \\
\text { McClelland (1990); Pathare et al. } \\
\text { (2010); Lorenz (2013) }\end{array}$ \\
\hline Morphology & $\begin{array}{l}\text { Columnar, disordered or v-shaped; } \\
\text { columnar vortices often include a v-shaped } \\
\text { 'sand skirt'; larger dust devils often include } \\
\text { sub-vortices }\end{array}$ & Metzger (1999) \\
\hline Rotation sense & Equally clockwise and counter-clockwise & $\begin{array}{l}\text { Flower (1936); Carroll and Ryan } \\
\text { (1970) }\end{array}$ \\
\hline $\begin{array}{l}\text { Wind speeds } \\
\text { (peak horizontal) } \\
\text { at } 2 \mathrm{~m} \text { height }\end{array}$ & $\begin{array}{l}\text { Usually peak at } 5-10 \mathrm{~m} \mathrm{~s}^{-1} \text {; recorded peak } \\
\text { winds of up to } 25 \mathrm{~m} \mathrm{~s}^{-1} \text { are not unusual }\end{array}$ & $\begin{array}{l}\text { Ryan and Carroll (1970); Balme } \\
\text { et al. (2003) }\end{array}$ \\
\hline $\begin{array}{l}\text { Wind speeds } \\
\text { (peak vertical) at } \\
1.0-4.5 \mathrm{~m} \text { height }\end{array}$ & $\begin{array}{l}\text { Usually } \sim 25 \% \text { of peak horizontal winds; } \\
\text { most peak measurements }<5 \mathrm{~m} \mathrm{~s}^{-1} ; \text { rare } \\
\text { values of } \sim 15 \mathrm{~m} \mathrm{~s}^{-1} \text { measured }\end{array}$ & $\begin{array}{l}\text { Sinclair (1973); Fitzjarrald (1973); } \\
\text { Metzger (1999); Metzger et al. } \\
\text { (2011) }\end{array}$ \\
\hline $\begin{array}{l}\text { Horizontal } \\
\text { translation speed }\end{array}$ & $\begin{array}{l}10-20 \% \text { greater than ambient wind speeds } \\
\text { measured at } 10 \mathrm{~m} \text { height; values of } \\
0--10 \mathrm{~m} \mathrm{~s}^{-1} \text { common; rare observations } \\
\text { of values }>20 \mathrm{~m} \mathrm{~s}^{-1}\end{array}$ & Balme et al. (2012) \\
\hline $\begin{array}{l}\text { Dust loading at } 2 \mathrm{~m} \\
\text { height }\end{array}$ & $\begin{array}{l}\text { Mean values (i.e., averaged across a profile } \\
\text { within each dust devil rather than peak } \\
\text { measurement) of } 0.8-42 \mathrm{mg} \mathrm{m}^{-3} \text { measured } \\
\text { for fine particles }(0-10 \mathrm{~mm} \mathrm{diameter}) \text {; mean } \text { values of up to } 6-875 \mathrm{mg} \mathrm{m}^{-3} \text { for total } \\
\text { suspended load; average values across }>20 \\
\text { dust devils reveal particle load of } \\
\sim 44 \mathrm{mg} \mathrm{m}^{-3} \text { for fine particles, } \\
\sim 300 \mathrm{mg} \mathrm{m}^{-3} \text { for total suspended load }\end{array}$ & Metzger et al. (2011) \\
\hline $\begin{array}{l}\text { Core temperature } \\
\text { excursion }\end{array}$ & $\begin{array}{l}\text { Wide range of temperature excursions } \\
\text { measured-probably due to variations in } \\
\text { sensor type; excursions of } 1-5^{\circ} \mathrm{C} \text { common; } \\
\text { larger excursions of }>20^{\circ} \mathrm{C} \text { reported }\end{array}$ & $\begin{array}{l}\text { Metzger (1999); Tratt et al. (2003); } \\
\text { Sinclair (1964); Sinclair (1973) }\end{array}$ \\
\hline $\begin{array}{l}\text { Core pressure } \\
\text { excursion (DP) }\end{array}$ & $\begin{array}{l}\text { Mobile sampling systems appear to give } \\
\text { larger values: } \sim 1-10 \text { mbar; fixed } \\
\text { monitoring stations give values }<1.5 \mathrm{mbar}\end{array}$ & $\begin{array}{l}\text { Sinclair (1973); Metzger (1999); } \\
\text { Lorenz and Lanagan (2014) }\end{array}$ \\
\hline Electric fields & $\begin{array}{l}\text { Field of } \sim 10-100 \mathrm{kV} / \mathrm{m} \text { measured; early } \\
\text { measurements hampered by field reaching } \\
\text { measurement limit of instrument }\end{array}$ & $\begin{array}{l}\text { Jackson and Farrell (2006); Renno } \\
\text { et al. (2004); Esposito et al. (2016) }\end{array}$ \\
\hline
\end{tabular}

relying on untended instruments can find it hard to distinguish between a population of long-lived dust devils and a population of more frequently occurring, but short lived dust devils.

A summary of observed characteristics is provided in Table 1. 
Fig. 2 (a) A digital camera image of a dust devil in Eloy, AZ in summer 2008. (b) A thermal image of the same devil a few seconds later with a FLIR Infracam hand-held thermal imager $(240 \times 240$ pixels $)$. Note that the image scale is not the same as in the optical image. The bar at the base of the thermal image indicates the brightness temperature grey scale in Celsius a)

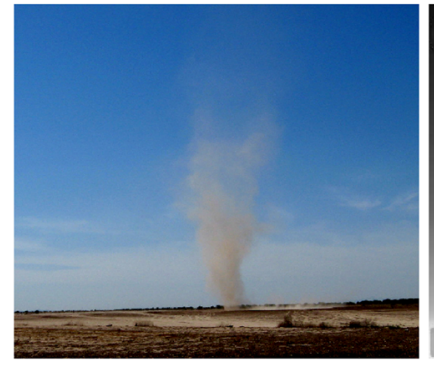

b)

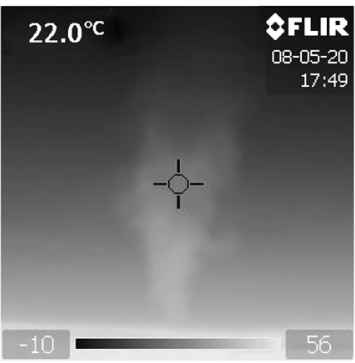

\subsection{Remotely Observed Terrestrial Dust Devil Characteristics}

\subsubsection{Morphology}

Dust devil morphology has been measured almost exclusively by surface based remote imaging observation (Figs. 1 and 2). As described by Lorenz et al. (2016), dust devils have been observed for many centuries and many authors describe them as "dusty-columns" or "upright whirlwinds". However, as shown by Metzger (1999), many dust devils do not have this 'classic' form; many are simply disordered clouds of barely-spinning dust, others are morerapidly spinning, v-shaped cones of entrained dust and sand, and others do have the wellknown, rapidly-spinning columnar shape. In a study in Nevada, USA, Metzger (1999) found that only about $4 \%$ of observed dust devils have a columnar shape. Even for those with columnar vortices, their morphology can differ: some have a clear core, others do not; some include a v-shaped "skirt" at their base, but others do not. Finally, some dust devils include sub-vortices that have their own centre of rotation but orbit the main circulation or which trail in their wake (e.g., Williams 1948; Ryan and Carroll 1970; Hallett and Hoffer 1971; Sinclair 1973; Metzger 1999). In many cases, the main circulation is barely dust-charged at all, so in these cases it is difficult to judge whether this is one large dust devil with subsidiary rotational elements, or a group of interacting smaller dust devils.

Most dust devils are higher than their widths. Hess and Spillane (1990) suggest that most are at least five times higher than their width, but again, many opposing observations exist. For those dust devils tall enough that a vertical structure can be observed, Sinclair (1966) provides a still-relevant summary: Region 1 is the zone nearest the ground, is heavily particle-loaded and often has a v-shaped form (Metzger 1999, refers to this as a "sandskirt"). Region 2, at intermediate height, is the near- vertical column of rotating dust. Region 3, aloft, is where the rotation decays and where the dust devils "fades" into the ambient atmosphere. These regions have been associated with different flow regimes (see, for example, Balme and Greeley 2006, Fig. 9): region 1 is where the majority of the radial inflow occurs, while Region 2 is characterised by rotation and uplift, and Region 3 has poorly characterised flows as here the structure dissipates.

\subsubsection{Size}

Dust devils on Earth range in size from a few metres in diameter and height to $100 \mathrm{~s}$ of metres in diameter and perhaps more than a kilometre in height. While estimates or measurements of diameter are relatively easy to obtain, determining dust devil height is more difficult, especially from surface observations. Also, it must be recognised that dust devil height refers 
to the observable dust column height, as opposed to the height of any circulation associated with the dust devil. For the visible column, estimates suggest that most dust devils are less than $50 \mathrm{~m}$ high, with only about $8 \%$ extending higher than $300 \mathrm{~m}$ (Sinclair 1965; Flower 1936; Williams 1948). However, observations made from the air identify taller dust devils that are 1-2.5 km in height (Bell 1967). Finally, Sinclair (1966) notes that measurable vertical wind speed and temperature excursions occur above large dust devils at heights of 2-4 km. However, it is not clear that these represent the upper parts of a dust devil per-se, or instead are associated with a broader circulation in which the dust devil is embedded.

The diameter-frequency of dust devils has been the subject of much study, with methodologies including simple "by-eye" observer surveys to more sophisticated studies that use time-lapse cameras or arrays of meteorology data. As noted by Lorenz (2011), most visual surveys (e.g., Sinclair 1965, 1969; Ryan and Carroll 1970; Snow and McClelland 1990) report that small dust devils are underreported by observers. Possible exceptions to this are the studies of Carroll and Ryan (1970), Pathare et al. (2010) and Balme et al. (2012), which both used small (1 km by $1 \mathrm{~km}$ or smaller) study areas. Several recent studies have focused on power law and other functional forms that best describe the dust devil size-frequency population (e.g., Kurgansky 2006; Lorenz 2009, 2011; Pathare et al. 2010)—a topic which is described in more detail by Lorenz and Jackson (2016) in this issue.

What is clear from all these studies is that the diameter-frequency distribution of dust devils is significantly skewed, with far more small dust devils occurring than large. Hence, the concept of an "average" dust devil diameter is not necessarily a useful one. What is clear, though, is that dust devils narrower than 5-6 meters in diameter are far more common than those wider than 10-12 $\mathrm{m}$, and that dust devils wider than $50 \mathrm{~m}$ are actually rather rare (e.g. Balme and Greeley 2006, Fig. 3; Lorenz 2011, Table 1).

\subsubsection{Translation Speeds}

Compared with Mars, few measurements of translational speed of terrestrial dust devils have been made. This is mainly due to the lack of top-down remote sensing of dust devils available for the Earth. Some ad-hoc measurements were made in the twentieth century (e.g. Crozier 1970) but the only focussed study, aimed solely at measuring the forward motion of dust devils, is that of Balme et al. (2012), who used stereo photography of dust devils to locate them in time and space. Multiple observations were made of each dust devil, thus allowing a path and thus a velocity to be calculated for each dust devil. In addition, as Balme et al. (2012) employed two $10 \mathrm{~m}$ high meteorology masts within their study area, they were able to correlate dust devil motion with ambient wind speed and direction. During the 10 days of field sampling, covering two calendar years and two study sites, translation speeds of between 1 and $15 \mathrm{~m} \mathrm{~s}^{-1}$ were measured for more than 100 dust devils.

Balme et al. (2012) found that dust devils translated in the same direction as ambient wind, and that the dust devil forward speed correlated well with ambient wind speed. In fact, they found that dust devils travel at about the same speed as the boundary layer winds a few tens of metres above ground. Interestingly, no correlation of translation speed with dust devil diameter was found. Therefore, Balme et al. (2012) conclude that dust devil forward motion is a good proxy for the wind field, and that dust devil motions is governed almost solely by local wind patterns - a finding that could prove to be important for Mars, where few meteorology data are available.

\subsubsection{Rotational and Vertical Speeds Within Dust Devils}

Measurement of the swirling winds within dust devils has generally been accomplished using in-situ sampling, though particle imaging velocimetry (Ito and Niino 2014) and re- 
mote measurements using LIght Detection And Ranging, or "LIDAR" (e.g., Schwiesow et al. 1977; Bluestein and Pazmany 2000) have also been employed. Obtaining statistically significant quantities of data is challenging for two main reasons. Firstly, even the most efficient sampling methodology (arguably, the mobile sampling platform approach) will only be able to sample a few dust devils per day, due to the short-lived and random nature of the phenomenon. Fixed sampling positions mean an even lower number of samples are likely, although this problem can be ameliorated by using many sensors in large arrays, or by sampling for very long periods of time. It is possible that dedicated LIDAR studies could also gather large quantities of data but to date no such attempts have been made. To date, the largest published study is that of Ryan and Carroll (1970), who sampled 80 dust devils. Another more recent large dataset exists (see preliminary report in Metzger et al. 2011) and includes more than 50 measurements from mobile in-situ sampling. However, these data are yet to be formally published. Aside from these two examples, individual studies usually report fewer than twenty encounters (Balme and Greeley 2006).

Both in-situ and remote sampling of wind speeds share the second problem-smaller and/or shorter-lived dust devils are harder to sample, and therefore are likely to be under represented in the data. This problem is likely to affect mobile "chase" strategies most significantly, as only the larger and longer-lived dust devils can be caught and penetrated to acquire data. Similarly, fixed-position remote-sensing studies are liable to target the most easily seen dust devils and could easily miss small, less dusty examples. The remaining methodology - that of fixed long-term meteorology stations-should be able to remove this sample bias, but again there is the issue of detectability, although this time the problem is how to tease out "detections" from the data. Significant progress has been made in this area recently; by using a single meteorology station and a theoretical dust devil 'signature', Lorenz (2016) was able to reconstruct peak wind speeds (and other signature parameters) and miss distance (i.e. the distance from the dust devil core to the sensor) for 27 dust devil events in 16 days of field time. However, the calculated diameters for these dust devils are all larger than $10 \mathrm{~m}$-suggesting that these too are "exceptional" events and again the more typical, smaller examples have not been detected.

Despite these caveats, the wind speeds within dust devils have been measured. Some authors have measured only the magnitude of the horizontal wind speeds within the dust devils, whereas others have provided all three components (i.e. inflow, tangential and vertical wind speed). Speeds are usually quoted at a height of $2 \mathrm{~m}$ above the ground, but measurements both nearer the ground (e.g. Balme et al. 2003) and higher into the dust devil (e.g. Kaimal and Bussinger 1970) have been reported. The following key points have emerged: (i) the horizontal winds within dust devils can often reach $10 \mathrm{~m} \mathrm{~s}^{-1}$ (e.g., Ryan and Carroll 1970), can peak at $>25 \mathrm{~m} \mathrm{~s}^{-1}$, but rarely, if ever, exceed $30 \mathrm{~m} \mathrm{~s}^{-1}$ (see Balme and Greeley 2006, Table 4, and Lorenz 2016, Table 1), (ii) vertical wind speeds are usually a factor of several less than the horizontal winds (e.g., Balme and Greeley 2006, Table 4), (iii) larger dust devils appear to contain stronger swirling winds, but vertical wind speeds do not correlate with diameter (e.g., Ryan and Carroll 1970), and (iv) the surface shear stress provided by the wind speeds within terrestrial dust devils appears sufficient to lift almost all sizes of naturally occurring loose sediments up to about granule-sized material (Balme et al. 2003).

\subsubsection{Dust Load}

Due to the fast-changing environment within dust devils, the concentrations of airborne dust and larger particles within dust devils are difficult to measure. The most complete study of particle loading in dust devils is that of Metzger et al. (2011), who used in-situ sampling 
on a mobile platform. Few other studies exist for terrestrial dust devils: a preliminary LIDAR observation at $100 \mathrm{~m}$ height (Renno et al. 2004) provided an estimate of dust load of $\sim 100 \mathrm{mg} \mathrm{m}^{-3}$ and there are reports of aircraft in-situ sampling of dust devils at 140 and $300 \mathrm{~m}$ height (Gillette and Sinclair 1990), but only flux data are given, not particle load. Other datasets exist for martian dust devils (e.g. Greeley et al. 2006, 2010), but Metzger et al. (2011) provide the main source of dust load data for Earth.

Metzger et al. (2011) present data from more than 30 encounters at two field sites and over four field seasons. They used both PM-10 sensors (sensitive to dust grade materials, 0.1-10 $\mu \mathrm{m}$ diameter) and total suspended load sensors (dust- to sand-grade materials). All measurements were made at the base of the dust devil (sensors were generally at $2 \mathrm{~m}$ height, but some measurements at 1,2.8 and $4.5 \mathrm{~m}$ height are reported). They found that PM10 dust load had high intra- and inter-dust devil variability. In many cases they report both maximum and mean dust load per dust devil (rather than just reporting the peak dust load) and report a peak range of $6-162 \mathrm{mg} \mathrm{m}^{-3}$ and mean range of $0.8-42 \mathrm{mg} \mathrm{m}^{-3}$. The measured total suspended particle load (i.e. including larger sediments) was much higher: ranging from 6-875 $\mathrm{mg} \mathrm{m}^{-3}$. Metzger et al. (2011) conclude that mean peak dust load (i.e. the amount of dust likely to lofted to height by a dust devil) is about three times less than the peak load measured, and that the total suspended particle load near the base of the dust devil is about ten time greater than the PM-10 fraction. They note, however, that the larger size fraction material is unlikely to be transported to great height, and is probably redeposited locally. This measurement is in agreement with observations of a "sand skirt" at the base of many dust devils. Oke et al. (2007) measured particle size within the bottom $\sim 1.5$ meters of willywillies, finding that sand sized particles were confined below $\sim 20$ centimeters. Raack et al. (2014) find similar results in Morocco.

\subsubsection{Seasonal and Diurnal Frequency of Occurrence}

As they are convective vortices, driven primarily insolation, dust devils occur most frequently when there is strong, continuous sunshine. This is usually in the summer, but they can occur at any time of year when there is a significant thermal contrast between the ground and the atmosphere (for example, dust devils have been seen in the Canadian sub-arctic; Grant 1949). To our knowledge, no season-to-season monitoring of dust devil activity has been performed to further refine the seasonal frequency, though.

In terms of diurnal rate of occurrence, many authors have noted that dust devils form most frequently in the late morning and the early afternoon (see summary by Balme and Greeley 2006 and recent work by Kurgansky et al. 2011). However, many of these reports are based on observer surveys which are both qualitative and which are unlikely to have run throughout the day-so there is always a possibility of bias. Recent work by Lorenz and Lanagan (2014) using a continuous month-long survey of pressure excursions to detect dust devils showed that most dust devils occur between 10:00 and 16:00 local time. They do note that about $10 \%$ of the day's dust devil events occur after 16:00 and there is a measurable tail of activity even after 18:00.

Several authors note that dust devil events are 'clustered' in time, with periods of more intense activity separated by periods of less activity. Carroll and Ryan (1970) suggest a periodicity of around 45 minutes and Renno et al. (2004) a periodicity of about 20 minutes. Lorenz and Lanagan's (2014) pressure-excursion data hint at a similar result.

In addition to surveys by human observers making either continuous records or recording at intervals (e.g. how many are seen at 15-minute intervals), the availability of time-lapse cameras, webcams etc. now allow new surveys with superior temporal coverage, and with 
quantitative detection criteria (e.g. optical contrast of $1 \%$ ). It is important in all such surveys that the detection criterion (size, contrast), and measurement cadence be documented-for instance images acquired at a given cadence or observing distance may preferentially detect a particular size of dust devils (Lorenz 2011, 2014; Kurgansky et al. 2011).

\subsubsection{Thermal Imaging}

The warm core of a dust devil and/or its suspended dust warmed by insolation absorption would be expected to provide a radiative thermal signature. Lorenz (2004) provided the apparent first scientific report of thermal infrared imaging of a dust devil (although Metzger et al. 2010, have since reported thermal imaging of Atacama dust devils, and Towner 2008, report orbital thermal imaging of dust devils at Mars). Thermal imagers have reduced significantly in cost in recent years, in part due to application in home improvement (to detect damp or poor insulation in walls). An example image is shown in Fig. 2.

The Lorenz (2004) observation reported a single dust devil as it moved away from an initial distance of 10 meters. The vortex temperature of $38-40{ }^{\circ} \mathrm{C}$ derived from the $8-14 \mu \mathrm{m}$ emission was approximately the same as the measured ambient air temperature but greater than the background atmospheric 'brightness' temperature of $12-20{ }^{\circ} \mathrm{C}$ obtained for lines of sight that did not intersect the dust devil. Lorenz (2004) noted that the dust might even be physically warmer than the surrounding air due to its interception of sunlight, a factor that might enhance the intensity of a vortex when dust is lifted (as later discussed by Fuerstenau 2006). Thermal imaging might provide a higher-sensitivity means of detecting marginally visible dust devils under some circumstances (notably, low-light levels) but this has not been robustly demonstrated. It may be that thermal imaging could also help visualize the nearsurface wind stress field around the devil (e.g. showing 'spiral' arms of the inflow, as can sometimes be seen on the ruffled surface of the sea around waterspouts) due to the winddependence of surface heat transfer.

\subsection{Remotely Imaged Martian Dust Devil Characteristics}

The opportunities for martian surface-based acquisition of visible imaging and subsequent characterization of dust devils has been limited to the two Viking Landers (1976-1982), Mars Pathfinder (1997), Mars Exploration Rovers Spirit (2004-2010) and Opportunity (2004-present), Phoenix Lander (2008), and Mars Science Laboratory Curiosity Rover (2012-present). Some of these missions provided no visible evidence of dust devil occurrence (Viking), while others provided the current best martian dust devil climatology (Spirit). Because imaging observations are discretely separated between robotic missions, we follow a chronological mission order presentation below.

A summary of observations is presented in Table 2.

\subsubsection{Viking Lander \& Mars Pathfinder Imaging}

The two Viking Landers safely settled onto Mars' surface during 1976, at subtropical (VL1) and middle (VL2) northern latitudes. VL1 returned measurements spanning 2245 sols covering portions of four martian years, while VL2 returned measurements for 1050 sols. Despite the substantial temporal extent of the Viking lander imaging data sets, these landers were unable to image dust devils because their cameras were facsimile-type imagers not well-suited to detecting moving objects (Lorenz et al. 2016). As a consequence of this characteristic of the Viking camera system, the first opportunity to visually detect martian dust devils from a 
Table 2 Martian dust devil observed characteristics

\begin{tabular}{|c|c|c|}
\hline Parameter & Best observations or measurements & Key literature \\
\hline Size (diameter) & $\begin{array}{l}\text { Inferred core diameters } 10-700 \text { meters from } \\
\text { Viking wind measurements (not visually } \\
\text { confirmed); } 15-550 \mathrm{~m} \text { (most frequent } 100-200 \\
\mathrm{~m} \text { ) from Pathfinder IMP (for assumed } 10 \mathrm{~m} \mathrm{~s}^{-1} \\
\text { translation speed); median diameter } 20-40 \mathrm{~m} \\
\text { from Spirit Nav Cam; MSL pressures and winds } \\
\text { ( } 16 \mathrm{~m} \text { median; } 21 \text { meter mean) }\end{array}$ & $\begin{array}{l}\text { Ryan and Lucich (1983); } \\
\text { Ringrose et al. (2003); } \\
\text { Ferri et al. (2003); } \\
\text { Greeley et al. (2010); } \\
\text { Kahanpää et al. (2016) }\end{array}$ \\
\hline Size (height) & $\begin{array}{l}\sim 10-400 \mathrm{~m} \text { (though many images truncate } \\
\text { vortex top); maximum verified lower limit } \\
\sim 800 \mathrm{~m}\end{array}$ & Greeley et al. (2006) \\
\hline $\begin{array}{l}\text { Lifetime/Detection } \\
\text { Duration }\end{array}$ & $\begin{array}{l}\text { 120-180 seconds from imaging; wind effect } \\
\text { determination duration ( } 60-1000 \text { seconds); } \\
\text { FWHM from wind pressure measurements } \\
\text { (5-20 seconds) }\end{array}$ & $\begin{array}{l}\text { Ringrose et al. (2003); Ferri } \\
\text { et al. (2003); Greeley et al. } \\
\text { (2010); Ellehoj et al. (2010); } \\
\text { Kahanpää et al. (2016); } \\
\text { Steakley and Murphy (2016) }\end{array}$ \\
\hline Morphology & $\begin{array}{l}\text { Columnar, disordered or v-shaped; few columnar } \\
\text { vortices include a v-shaped 'sand skirt' }\end{array}$ & $\begin{array}{l}\text { Greeley et al. (2006); } \\
\text { Ferri et al. (2003) }\end{array}$ \\
\hline Rotation sense & Equally clockwise and counter-clockwise & Ryan and Lucich (1983) \\
\hline $\begin{array}{l}\text { Wind speeds (peak } \\
\text { horizontal) at } \\
\sim 2 \mathrm{~m} \text { height }\end{array}$ & $\begin{array}{l}\text { Maximum measured speeds are } \sim 30-40 \mathrm{~m} \mathrm{~s}^{-1} \text {; } \\
\text { maximum inferred core boundary speeds } \\
\text { approach } 100 \mathrm{~m} \mathrm{~s}^{-1} \text { for two instances which } \\
\text { correspond with the greatest spatial } \\
\text { extrapolation to that core boundary position }\end{array}$ & $\begin{array}{l}\text { Ryan and Lucich (1983); } \\
\text { Ringrose et al. (2003); } \\
\text { Ellehoj et al. (2010) }\end{array}$ \\
\hline $\begin{array}{l}\text { Wind speeds (peak } \\
\text { vertical) from } \\
\text { imaging }\end{array}$ & $\begin{array}{l}\text { Maximum } \sim 17 \mathrm{~m} \mathrm{~s}^{-1}, \text { with median } \\
1.0-1.6 \mathrm{~m} \mathrm{~s}^{-1}\end{array}$ & Greeley et al. (2010) \\
\hline $\begin{array}{l}\text { Horizontal } \\
\text { translation speed }\end{array}$ & $\begin{array}{l}\text { A few to } \sim 25 \mathrm{~ms}^{-1} \text { (median } \sim 2 \mathrm{~m} \mathrm{~s}^{-1} \text { ) (Note: } \\
\text { ambient wind speeds were not correspondingly } \\
\text { available) }\end{array}$ & Greeley et al. (2010) \\
\hline Dust loading & $\begin{array}{l}700 \mathrm{mg} \mathrm{m}^{-2} \text { for a horizontal path through a dust } \\
\text { devil, implying } 20 \mathrm{mg} \mathrm{m}^{-3} \text { for a } 35-\mathrm{m} \mathrm{diameter} \\
\text { dust devil, for Pathfinder; } 0.002-250 \mathrm{mg} \mathrm{m}^{-3} \\
\text { for Spirit; coarse particles ( }>63 \text { micrometers) } \\
\text { confined to }<30 \mathrm{~cm} \text { above the surface }\end{array}$ & $\begin{array}{l}\text { Metzger et al. (1999); } \\
\text { Greeley et al. (2010); } \\
\text { Oke et al. (2007) }\end{array}$ \\
\hline $\begin{array}{l}\text { Temperature } \\
\text { excursion }\end{array}$ & $\begin{array}{l}\text { Measured excursions of } 1-6^{\circ} \mathrm{C} \text { for Viking \& } \\
\text { Pathfinder \& Phoenix; MSL/REMS }\end{array}$ & $\begin{array}{l}\text { Ryan and Lucich (1983); } \\
\text { Murphy and Nelli (2002); } \\
\text { Ringrose et al. (2003); } \\
\text { Ellehoj et al. 2010); } \\
\text { Kahanpää et al. (2016) }\end{array}$ \\
\hline $\begin{array}{l}\text { Core pressure } \\
\text { excursion }(\Delta P)\end{array}$ & $\begin{array}{l}0.3 \text { to } \sim 5 \mathrm{~Pa} \text {; lower limit arises from limit } \\
\text { imposed upon the analyses, with smaller } \\
\text { magnitudes more frequent; no measurement } \\
\text { regarding verified vortex maximum } \Delta P \\
\text { excursion is available }\end{array}$ & $\begin{array}{l}\text { Murphy and Nelli (2002); } \\
\text { Ellehoj et al. (2010); } \\
\text { Kahanpää et al. (2016); } \\
\text { Steakley and Murphy (2016) }\end{array}$ \\
\hline Electric fields & No measurements available & \\
\hline
\end{tabular}

surface vantage point occurred with the Mars Pathfinder Lander (MPF) mission. It is tempting to speculate that the facsimile-type imager on the Viking landers may have a few dark lines in parts of a few images caused by dust devil passages during their slow scans, but these would be challenging to detect and attribute. 
MPF began operation on Mars' surface on 04 July, 1997 in the northern subtropical (19.3 N, 33.4 W; Golombek et al. 1997) Ares Vallis region located 1000 kilometers eastsoutheast of Viking Lander 1. The mission provided measurements spanning 83 sols covering the latter third of northern summer through very early northern autumn $\left(L_{s} 142-183\right)$. There was some expectation that Pathfinder would have the opportunity to detect dust devil signatures within its meteorology (MET) measurements (Seiff et al. 1997).

From analysis of multi-color images provided by the Imager for Mars Pathfinder (IMP) (Smith et al. 1997), Metzger et al. (1999) reported the first identification of martian dust plumes within surface-obtained images. Image enhancement via band subtraction was employed to isolate the low-contrast signature of the dust plume. Dust plumes were most evident as occultation features at blue $(430 \mathrm{~nm})$ wavelengths among the Imager for Mars Pathfinder (IMP) wavelength filters, which also included 530 and $670 \mathrm{~nm}$, due to the reduced dust scattering at that shorter wavelength. Five dust plumes were identified within 16 analyzed IMP images obtained near mid-sol on sols 10 and 11 of the 83-sol mission when landing site panoramas were being acquired. Dust devil diameter (14-79 meter) and height (46-450 meters) and translation speed $\left(0.5-4.6 \mathrm{~m} \mathrm{~s}^{-1}\right)$ were estimated from the angular width and motion derived from multiple images and the inferred distance from the lander obtained from identification of foreground and background features. The plume/vortex dust load $\left(\sim 7 \times 10^{-5} \mathrm{~kg} \mathrm{~m}^{-3}\right)$ was estimated to be $\sim 10,000$ times greater than the background dust load.

Ferri et al. (2003) applied the Metzger et al. (1999) band subtraction technique to a more extensive set of horizon-containing IMP images and identified 14 dust plumes/devils that included the 5 from Metzger et al. (1999). Only one of these identifications occurred for an image that was not part of the panorama captured during mission sols 10-11. To quantify vortex size, a constant translation speed of $10 \mathrm{~m} \mathrm{~s}^{-1}$ was assumed, with the observed angular rate of motion from consecutive images being employed to estimate vortex distance and subsequently vortex size. The 14 identified vortices were estimated to span the size range of 10-570 meters and to have been positioned 1-25 km from the lander. A surface vortex surface area coverage of $2 \times 10^{-4}(0.02 \%)$ was estimated for the 0900-1500 local true solar time (LTST) time interval. This fractional coverage, coupled with a derived vortex vertical dust flux estimate of $7 \times 10^{-5} \mathrm{~kg} \mathrm{~m}^{-2} \mathrm{~s}-1$, resulted in an estimated total vortex-induced vertical dust flux of $3.6 \times 10^{-9} \mathrm{~kg} \mathrm{~m}^{-2} \mathrm{~s}^{-1}$, which exceeded by an order of magnitude the estimated local dust deposition rate (Landis and Jenkins 2000).

In addition to images, one IMP-provided opacity measurement from direct solar imaging on sol 14 resulted in larger values at all wavelengths compared to more than 10 additional opacity observations obtained that same sol (Smith and Lemmon 1999). This increased opacity event could possibly have been the result of a dust-laden vortex occulting the Sun but no additional supporting measurements, including contemporaneous MET measurements, are available.

Thus, the Pathfinder mission did verify that dust devils, or at least dust plumes (since motion within a plume was not identified), are visible from Mars' surface, but these imaging results did not provide a rigorous quantitative characterization of martian dust devils.

\subsubsection{Mars Exploration Rovers (Spirit \& Opportunity)}

After the experience gained with the martian dust devils identified in the MPF IMP images, the Mars Exploration Rover (MER-A \& B-Spirit \& Opportunity) science teams prepared for the possibility of observing dust devils at the two landing sites: Gusev Crater (Spirit) and Meridiani Planum (Opportunity). MER science teams planned on using similar band-subtraction techniques for viewing dust devils as was originally used by Metzger 
Fig. 3 Three images from a typical Spirit-Navigation camera sequence used to create martian dust devil "movies". Numbers in the lower left of each image indicate the number of seconds since the image sequence began. This sol 456 dust devil viewed from the west flank of the Columbia Hills translates from left to right, passing over a small bright toned depression as it moves

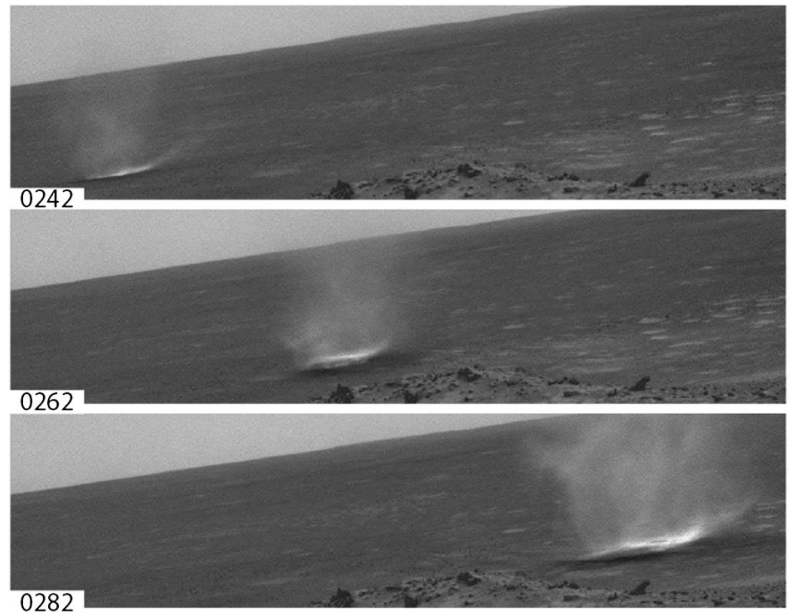

0282

et al. (1999) to enhance the contrast to make these difficult phenomena easier to study. The Panorama Cameras (Pancams) on the MERs were higher resolution than the Imager for Mars Pathfinder (IMP) and capable of better images, but it was not known if either of the two locations would be capable of producing better, more visible dust devils than those observed in Ares Vallis with MPF. Orbital images suggested that Gusev Crater (Spirit) would have a good chance of seeing dust devils because of a swath of features across the crater with many dust devil tracks (Greeley et al. 2003). However, prior to Spirit's landing, active dust devils had not been observed from orbit in Gusev crater, casting doubt that the swath of dark features were in fact dust devil tracks. Spirit landed near the end of Southern Hemisphere Summer ( $\left.\sim L_{s} 330\right)$ [Squyres et al. 2004]. The first part of the mission produced no imaging-detected dust devils at either location for the rovers, and many searches through images using the band subtraction technique yielded no results (Lemmon et al. 2004), although one new dust devil track was observed from orbit, having formed between $L_{s} 12$ and 22 (Lemmon et al. 2015). On sol 421 ( $L_{s} 173$ ), while Spirit was perched on the Columbia Hills near the center of the Gusev, the first dust devil was observed, differing greatly from the nebulous wisps from the MPF images. The MER image was crisp and detailed and as with subsequent observations, the dust devils were clearly visible as distinct from the background (Fig. 3). Observations at Meridiani by Opportunity have been limited to a few sightings of individual dust devils that could be a result of poorer viewing geometry and/or limited liftable dust (Lemmon et al. 2015).

After the first year of the mission, an elevated vantage point on Husband Hill offered several advantages over the previous lander geometry (Greeley et al. 2006). Sitting above the plains of the crater floor, the dust devils appeared bright against the ground, and darker against the sky above the horizon. The elevated viewing angle also allowed each dust devil to be more precisely located against surface features such as smaller craters and hollows, and rock patterns. More precise locations allowed better distances to be known, allowing for better estimations of sizes. Once the first dust devil season officially started, it became clear that dust devils could be seen easily in all of the rover's camera systems including not only the Pancam, but also the lower resolution monochrome Navigation Camera (Navcam) and both the forward and rear Hazard Cameras (Hazcams). Specific imaging campaigns were designed to make use of what was known about dust devil statistics. Initial dedicated imaging occurred during 0900-1700 LST. Subsequently, Navcam and Pancam images 
were subframed upon the ground/sky boundary and the most common locales for dust devil occurrence. Subframing reduced the amount of storage space for each image and allowed multiple image, "movie", sequences to be obtained (Fig. 3). The $\sim 20$ second frame rate on the movie sequences was limited by the refresh rate of the cameras' CCDs and the image buffer, but the image acquisition times refresh cycle was well known, allowing time between frames to be accurately established. Due to the favorable viewing geometry, on many sols not only sizes of and distances to the dust devils could be determined, but also translation speeds (horizontal) of the dust devils suggesting background ambient wind speeds in Gusev. In some movie sequences, detail was high enough that pockets of dust from some of the hollows could be followed from frame to frame allowing rough estimates of vertical velocities to be determined.

The longevity of the MER campaigns allowed for repeat seasonal studies of dust devil activity, which was particularly useful in Gusev Crater. Spirit had observed $\sim 533$ dust devils in the first documented dust devil season (Greeley et al. 2006). This high number of individual vortices was due in part to excellent viewing geometry from atop the Columbia Hills, from where much more of the crater floor of Gusev was visible. As Spirit's traverse led it further south into the saddle and eventually to the "Home Plate" feature, Spirit's view of the crater floor was obstructed by the hills. Greeley et al. (2010) describes the three total observed seasons in detail. The second dust devil season began on about sol 1101 $\left(L_{s} 181^{\circ}\right)$, which was comparable to the first season's start around sol $421\left(L_{s} 173.2^{\circ}\right)$. The second dust devil season was truncated by the onset of a set of planet-encircling dust storms that restricted insolation at the surface, presumably prohibiting the formation of dust devils while the background atmospheric dust opacity rose to a peak tau of 4.31 (Greeley et al. 2010; Lemmon et al. 2015). While the dust opacity was so high, solar power for rover operations was limited and fewer images were taken, but of the images that were acquired, no dust devils were observed during the dust storm and the upper limit for dust devil frequency was an order of magnitude below pre-storm levels (Lemmon et al. 2015). The second season, with limited viewing geometry and the presence of strong regional dust storms, produced an observed 101 individually identified dust devils. The following martian year, 127 more vortices were observed when the third dust devil season began around sol $1785\left(L_{s} 189^{\circ}\right)$. Still located near Home Plate, Spirit's view of the plains was still restricted similarly to the second season (Fig. 4). Over three Mars years, Lemmon et al. (2015) found that changes in dust devil frequency correlated with changes in surface insolation, whether the changes were seasonal or from dust storms, and that dust devil frequency fell to $1 / e$ with each $18 \mathrm{~W} \mathrm{~m}^{-2}$ reduction in mean insolation (roughly $10 \%$ of the peak insolation) (Fig. 5).

Spirit and Opportunity were not equipped with meteorological instrument packages for temperature and pressure measurements, but over the three dust devil seasons recorded at Gusev, several key measurements were made using the camera systems. For the first time on Mars there was repeat coverage at the same site for dust devil seasonal information. Correcting for sample bias, Greeley et al. (2010) estimated that the diurnal distribution of dust devils at Gusev began after 0900 and tailed off before 1700 LTST. This time range was consistent for all three seasons at Gusev (Fig. 6). Peak activity occurred around 1300 LTST with some indication of possible burst of activity near the end of the day between 1400 and 1600 LTST. Tracking dust devils within movie sequences yielded estimates of both translational and vertical velocities. Translational speeds, which could serve as a rough surrogate to the background boundary layer winds across Gusev Crater were estimated to be between a few meters per second up to $\sim 27 \mathrm{~m} \mathrm{~s}^{-1}$ and maximum velocities tended to occur near the end of local springtime. Minimum vertical wind speeds 
Fig. 4 (After Greeley et al. 2010) Three seasons of dust devil occurrence locations within Gusev Crater. (a) Mosaic of HiRISE images of Spirit's operation area, with vectors indicating dust devil tracks from Spirit Navigation camera 'movies', color coded for each season (year); stars indicate locations of active dust devils from single frames. (b) Mars Orbiter Camera Wide Angle red image R21-00168 inset of Gusev Crater showing the Gusev Low Albdeo Zone (GLAZ, outlined) where dust devil tracks are observed, and the location of Fig. 4a
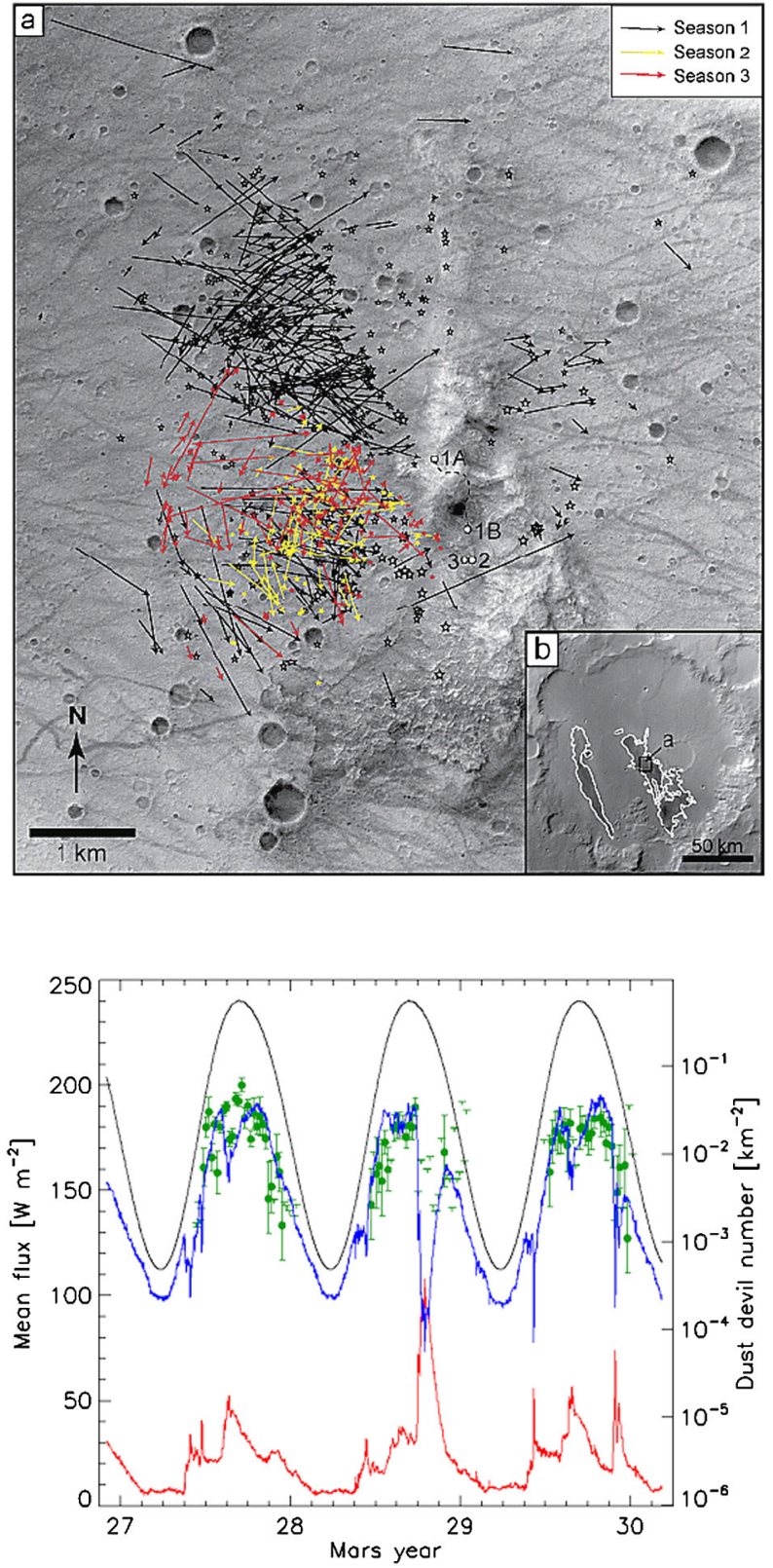

Fig. 5 Insolation at the Spirit rover site. The continuous curve shows the modeled

top-of-atmosphere, sol average insolation (upper, black), direct plus diffuse surface insolation (middle, blue) and atmospheric absorption of sunlight (lower, red). Symbols (green) show dust devil number density (right axis) reported by Greeley et al. (2010) with the $\mathrm{T}$ symbols indicating upper limits). (For the interpretation of the references to color in this figure legend, the reader is referred to the web version of this article)

within a few dust devil columns were estimated based on visual identification of small clumps of dusty material present from frame to frame in the movie sequences. Vertical wind speeds were estimated to be 0.04 up to $\sim 17 \mathrm{~m} \mathrm{~s}^{-1}$ with medians being between 1.0 and $1.6 \mathrm{~m} \mathrm{~s}^{-1}$. The data from the MER rovers, and Spirit in particular provide the most complete observation of a dust devil season on Mars and rival any campaign attempted on Earth. 
Fig. 6 (After Greeley et al. 2010) Number density of Spirit observed dust devils versus Local True Solar Time (LTST) for three seasons (years). $N$ is the total number of dust devils observed each season
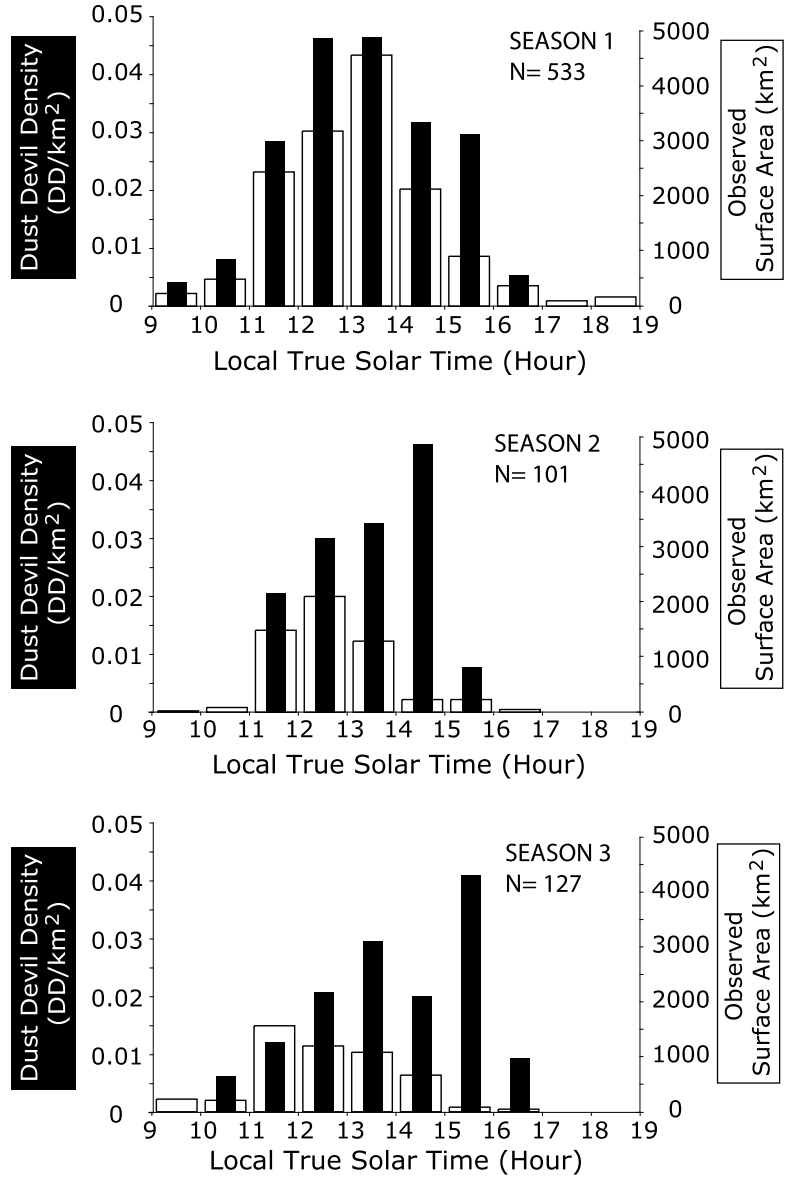

\subsubsection{Mars Phoenix Lander}

NASA's Mars Phoenix spacecraft (Smith et al. 2008, 2009) landed at an arctic location $\left(68.2^{\circ} \mathrm{N}, 234.3^{\circ} \mathrm{E}\right)$ in the Martian Northern Plains on 25 May 2008, in early northern hemisphere summer $\left(L_{s} 77^{\circ}\right)$. The primary mission lasted for 90 sols; contact was lost after 151 sols (circa 5 months), in late summer $\left(L_{s} 148^{\circ}\right)$. Equipped with the Surface Stereo Imager (SSI), with adequate resolution to image dust devils, and a high-resolution pressure sensor, Phoenix became the second Mars lander, after Pathfinder, that had the capacity to detect dust devils both visually and by meteorological measurements. It was, however, not until sol $104\left(L_{s}=125^{\circ}\right)$ that the first dust devil was spotted by SSI (Ellehoj et al. 2010). However, there had not been many opportunities to detect dust devils before this. Besides imaging geological targets close to the lander, this camera was used to monitor the Telltale wind indicator (Holstein-Rathlou et al. 2010). The first dust devil was detected serendipitously while imaging a panorama. After this first detection, Phoenix was commanded to take image sets aimed to search for dust devils. These sets consisted of 13 to 50 sequential images of the horizon.

Ellehoj et al. (2010) investigated the images taken by Phoenix of dust devils. Image contrast was enhanced, as had been previously done with the Pathfinder (Metzger et al. 1999; 
Fig. 7 This two-minute and 37-second time sequence (top-to-bottom) of contrast-enhanced Phoenix SSI images shows the translation of a mid-afternoon dust devil seen southwest of the lander on the mission's 109th sol $\left(L_{S}=127\right)$
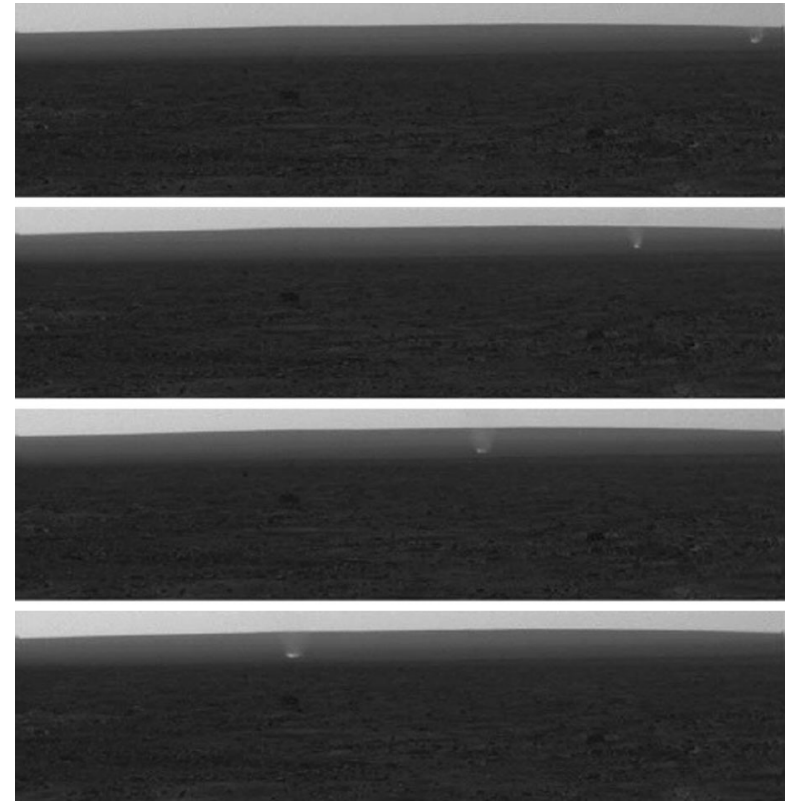

Ferri et al. 2003) and MER images (Greeley et al. 2006, 2010), allowing the detection of features with only an approximately $3 \%$ difference compared to background albedo. 37 individual dust devils were identified in the SSI images obtained between sol 104 and sol $138\left(L_{s}=125-142\right)$ (Fig. 7). The beginning and end of the Phoenix "dust devil season" could not be determined because the dust devil imaging campaign lasted only for a small fraction of the Martian year. However, the first Phoenix observations of dust devils were shortly (14 sols) after the Sun set for the first time in the mission - still near peak northern summer insolation, as at Gusev crater for southern summer, but when temperature contrasts could develop through diurnal cycles. The diurnal time range of SSI dust devil observations spanned 11:00 to 16:00 LTST, matching results of MER Spirit and the timing of vortices detected by the Phoenix pressure sensor (Sect. 3), although no dust devil search imaging was performed in the morning hours before 11:00 due to operational constraints on available energy to heat and aim the camera for use.

All dust devils were seen in the southwest direction, despite two-thirds of the horizon images having been obtained from other directions, with unobstructed views in all azimuths. The distances of the dust devils from the lander could not be determined in the featureless landscape so their physical size could not be evaluated. In most cases the dust devil was visible in several sequential images and was thus seen to move across the frame. As the distances were unknown, translation speeds could not be assessed. In most cases the direction of motion had a an eastward component, agreeing with the wind directions measured by the Telltale wind indicator and orbital observations of dust devils and their tracks in the same area (Holstein-Rathlou et al. 2010; Reiss et al. 2014). The dust devils detected by Phoenix were too faint to enable determination of vertical wind speeds as had been done for some of the dust devils imaged by MER Spirit (Ellehoj et al. 2010). 


\subsubsection{Mars Science Laboratory/Curiosity Rover}

The US/NASA Mars Science Laboratory (MSL) Curiosity rover has performed meteorological measurements since August 2012 in 154 kilometer diameter Gale crater, centered just south of the Martian equator (4.6 $\left.{ }^{\circ} \mathrm{S}, 137.4{ }^{\circ} \mathrm{E}\right)$. Studying the modern Martian environment is one of the science goals of NASA's Mars Science Laboratory (MSL) mission (Grotzinger et al. 2012). MSL is, unlike the MERs, equipped with a meteorological station, a video-capable color science camera, and a MER-like high signal-to-noise navigation camera system with a reasonably high frame rate. With this payload and a planned operational lifetime of more than one Martian year, MSL could have become an almost perfect lander for the study of dust devils. However, the landing site in Gale crater turned out to be less than ideal for this purpose.

Prior to MSL's landing it was expected that very few, if any, dust devils would occur at this site. No dust devil tracks had been seen in orbital images of the crater floor (Fred Calef, Jet Propulsion Laboratory/Caltech, personal communication, 2012). Further, atmospheric modeling (Tyler and Barnes 2013; 2015) suggested that the depth of the daytime boundary layer inside the crater is suppressed. This suppressed, shallow depth was expected to reduce vortex activity, or at least vortex intensity, since the thermodynamic efficiency of vortices depends upon the boundary layer depth according to the so-called heat engine model (Renno et al. 1998, 2000). Nevertheless, an extensive campaign of imaging Dust Devil Search Movies was initiated after MSL's landing (Moores et al. 2015). The Dust Devil Search Movies are taken using MSL's monochrome Navigation Cameras (Navcam) and consist of four to eight frames with the central elevation on the horizon. All together 91 Dust Devil Search Movies were imaged during the first 360 sols of the mission. The result matched the expectations: only one very faint dust devil was detected on sol $41\left(L_{s}=173\right)$. This virtual non-detection, combined with MSL's meteorological measurements, can be used to constrain the conditions where dust devils can form on Mars (Kahanpää et al. 2016; Klose et al. 2016).

Collectively, five spacecraft equipped with cameras suitable for imaging moving objects have landed on Mars: Mars Pathfinder, MER Spirit, MER Opportunity, Mars Phoenix and MSL. Despite the different landing sites, spanning latitudes from $14.6{ }^{\circ} \mathrm{S}$ (Spirit) to $68.2^{\circ} \mathrm{N}$ (Phoenix), all of these landers have succeeded in imaging dust devils (or at least dust plumes), indicating that dust devils occur on all latitudes on Mars. While the other landers have imaged from tens to hundreds of dust devils, MER Opportunity and MSL have observed only a few, in spite of the long durations of these missions and active search for dust devils, showing that there are strong variations in local dust devil occurrence rates.

While martian surface obtained imaging has provided direct evidence for dust devils, only the Spirit rover within Gusev Crater has provided measurements from which internal vortex characteristics have been quantified and then only minimally. The Spirit observations did provide valuable seasonal and time-of-sol occurrence characterization; Phoenix provided some seasonal indication of occurrence variation but the number of images available for analysis were more limited than the number and seasonal extent available from Spirit.

\section{Surface Obtained Meteorology Measurements of Dust Devils}

In addition to their visual manifestation, dust devils can also be characterized via in situ measurements of their thermodynamic conditions, including the central pressure drop, wind speed, temperature, suspended dust load, surface dust lifting magnitude, electrostatic state, 
etc. For such measurements to be of statistical characterization value, high sampling frequency and long duration (seasonal, annual) measurements are best, but until recently such long lived measurement opportunities were not the norm. Previous in situ measurements have provided much of the knowledge currently available regarding dust devil thermodynamics. Despite their often incomplete coverage and potential biases these measurements are the foundation upon which current and future measurements of terrestrial dust devils are constructed.

A compilation of measured characteristics is provided in Table 1.

\subsection{Meteorological Measurements of Terrestrial Dust Devils}

Sporadic serendipitous meteorological encounters with dust devils were reported in the early literature, such as an encounter with the barograph at a small airport (Wyett 1954) but the first systematic measurements began in the 1960s. A particular challenge is that dust devil phenomena generally occur on timescales (seconds) that require fast instrument response and data acquisition. Sinclair (1966) made pressure, temperature and wind measurements with a hand-carried recording instrument station, and later a more elaborate station mounted on a jeep. The mobile sensor platform allowed penetration of dust devils within a reasonably short 'hunting' season, and data acquisition arrangements included a cine camera recording instrument readings.

Also in the 1960s, two sets of fixed-station investigations were performed. Lambeth (1966) set up an array of 6 meteorology stations at White Sands Missile Range, and recorded (with chart recorders) 19 encounters in a several month period. This rather low encounter rate proved discouraging, leading the author to recommend vehicle-borne measurements. On the other hand, Ryan and Carroll (1970) made temperature and wind measurements at a single fixed mast in the Mojave desert, but groomed the ground around the mast to ensure dust availability.

Field studies of dust devils saw a renaissance in the late 1990s and early 2000s with the observation of dust devils on Mars by Mars Pathfinder (Sects. 2.2.1, 3.2.2), and the prospects for observing more there with a lander planned for 2001 (later cancelled) and by Beagle 2. These impending Mars missions lead to several field campaigns in Arizona and Nevada (e.g. Tratt et al. 2003; Ringrose et al. 2003; Renno et al. 2004; etc.) during which, again, the measurements were principally vehicle-borne.

The presentation below of terrestrial dust devil measurements follows a measured parameter structure, with emphasis upon more recent measurements.

\subsubsection{Pressure Measurements}

While providing a time-efficient means of acquiring measurements in dust devils, vehicleborne chase measurements do not reproduce how measurements are acquired on Mars, where a single fixed station records data over an extended time, but not continuously. In addition to vehicle disturbance of measurements (e.g. visible in Tratt et al. 2003) and often rather poorly-documented distance histories, the tendency to chase the biggest, slowest devils leads to strong selection biases, which make it difficult to estimate the characteristics of the dust devil population. Technological developments in flash memory in the late 2000s allowed inexpensive data acquisition (Lorenz 2012b) with compact and low-power systems that could be deployed for months, sampling at $>1 \mathrm{~Hz}$ without requiring operator visits to download data or replace power supplies. Furthermore, when only pressure and light levels are recorded, the logging package can be placed in 


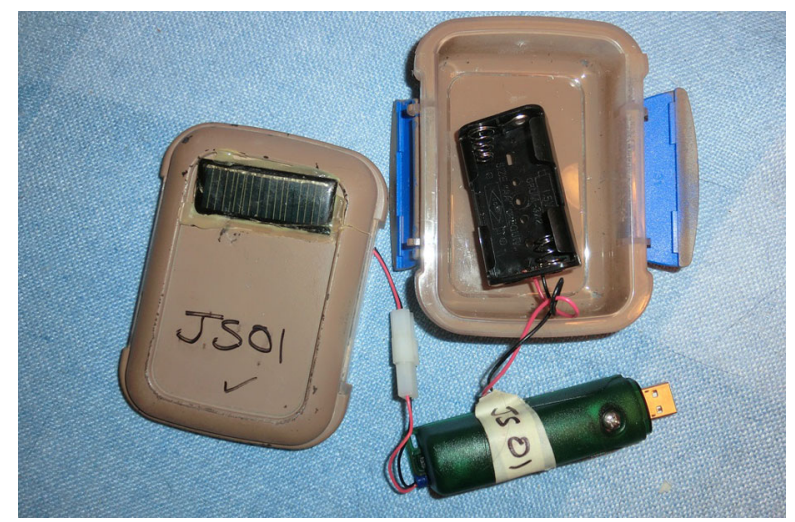

Fig. 8 Pressure and solar-flux logger used by Lorenz and Jackson (2015) and elsewhere. The commercial logger itself is essentially a USB memory stick (green cylindrical object at lower right) which can accommodate a single AA battery to operate for several days. Here a $2 \times$ AA battery holder (which due to peculiarities of the power supply system yields about a month of operation at $2 \mathrm{~Hz}$ ) is included, as well as a solar cell to record the shadow of dust devils. The whole unit can fit in a pocket. Note the logger identifier, necessary as these loggers have been installed in arrays

a box with a volume of less than 0.5 liters (Fig. 8) and simply set on the ground: this can be done in such a way (with a camouflaged housing) such that attrition by theft or vandalism at open sites is minimal. The systems are inexpensive enough $(\sim \$ 150)$ compared with the cost of deployment and retrieval that they can be considered somewhat expendable. These systems have allowed large numbers (hundreds to thousands) of unperturbed vortex encounters to be obtained without chase biases, finally yielding robust statistics on vortex populations from pressure drops (Lorenz and Lanagan 2014; Jackson and Lorenz 2015) and on dust loading (Lorenz and Jackson 2015). Furthermore, the small measurement stations can be deployed in spatial arrays to make simultaneous measurements that resolve the two-dimensional horizontal structure of dust devils (Lorenz et al. 2015a, 2015b, 2015c).

The pressure loggers developed by Lorenz (2012a, 2012b) are compact enough that they can be easily carried in a pocket and operate without attention for many days, allowing opportunistic pressure measurements during other field activities without extensive preparation. For example, Lorenz and Radebaugh (2016) report the first in-situ measurements of vortex activity at a high-elevation site (a yardang and gravel-ripple field at $3800 \mathrm{~m}$ in the Argentinian Andes high plain-Puna) using such methods, indicating higher levels of dust devil activity than reported previously elsewhere (see also Chap. 8 of this volume, Lorenz et al. 2016).

Pressure drops associated with dust devils have been recorded opportunistically at a few sites (notably by chart-recording barographs as early as the 1950s-see Chap. 1 of this volume, Lorenz et al. 2016). A number of pressure traces were obtained in vehicle encounters by Sinclair (1969) and subsequent studies, but until recently, the pressure signatures of vortex encounters were better-documented at Mars (Lorenz 2012a) than on Earth. The 'expendable' small pressure loggers advanced by Lorenz (2012b) were deployed at Eldorado playa near Boulder City, NV, and showed promise. Three such loggers were operated for a one-month period in June 2012 (Lorenz and Lanagan 2014), generating the first statistically-robust terrestrial dataset of fixed-station encounters (and allowing comparison of their power-law statistics with those at Mars—see Lorenz et al. 2016, in this issue). Note 

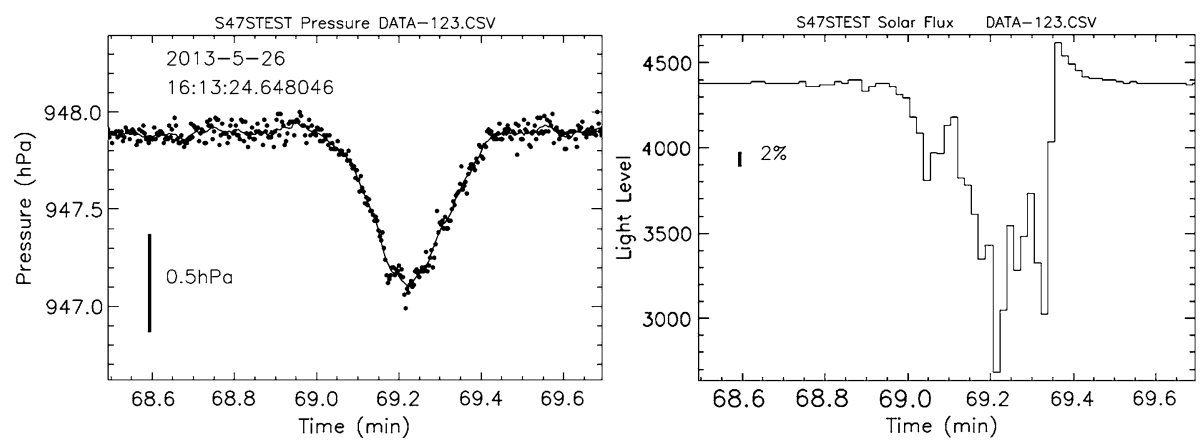

Fig. 9 A solar logger encounter with a large dust devil on Eldorado in summer 2013 (from Lorenz and Jackson 2015). A broad pressure drop lasting about $20 \mathrm{~s}$ is seen; this pressure drop of about 0.7 mbar is typically encountered not more than once in a few weeks. The solar flux measurement at right shows that the devil was heavily dust-laden, blocking about $30 \%$ of the light: the two prominent troughs are probably the wall of the dust column. Note that the light level *rises* after the encounter-part of the normally dark sky is occupied by dust which scatters light onto the solar cell in addition to the direct solar beam which is now no longer shadowed. This indicates the devil was moving away from the solar azimuth

that the recorded pressure drop at a point is a function of the vortex pressure field (related to diameter and intensity—see the companion chapter by Kurgansky et al. 2016) and the trajectory of the dust devil relative to the measurement station. Importantly, the data were made available for the use of other workers.

Jackson and Lorenz (2015) extended the Eldorado study with observations from several sites over summers 2012 and 2013, and the intervening winter, giving insights into the seasonal variation of vortex occurrence, and interannual variability (e.g. due to dust availability-flooding of the playa altering the surface texture and thus the lifting threshold—-see Neakrase et al. 2016 in this issue). That study also explored automatic detection methodologies (finding over 1000 events), since these measurement approaches develop many Gigabytes of data, for which the manual vortex detection employed by Lorenz and Lanagan (2014) would have been prohibitive: the $\sim 1200$ station-days of data comprise some 120 million measurements.

Lorenz and Jackson (2015) performed another study at 4 locations at Eldorado in summer 2013, using loggers with a solar cell to record dust devil shadows (Fig. 8). This study found that about half of pressure encounters were accompanied by measurable light level drops (Fig. 9). Some dustless vortices occurred, and in others, the devil's shadow missed the logger.

The Eldorado site is an open area, used for various recreational purposes, at which large unattended installations may encounter human interference (although artfully-concealed small loggers have been generally unaffected, although occasionally damaged by flooding). Lorenz et al. (2015b) employed another field site, La Jornada Experimental Range in New Mexico (operated by the US Department of Agriculture) where access is restricted and so larger installations can be left safely unattended.

This facility was used by Lorenz et al. (2015b) to deploy a line array of pressure/solar loggers in summer 2013. In addition to providing population statistics at this site, the survey noted that the number of vortices encountered varied quite substantially over a distance of a few tens of meters, due presumably to the influence of topography on dust devil migration and/or the effects of different scrub bushes on dust availability and surface roughness. This array study generated for the first time (since a pioneering chart-recorded 6-station study by 
Lambeth 1966) simultaneous measurements exposing the horizontal pressure structure of dust devils. An example dataset is shown in Fig. 10, illustrating the radial variation of the measured pressure drop detected as the vortex passed across the pressure sensor array.

\subsubsection{Wind}

In a supplemental investigation to pressure logging, Lorenz (2016) obtained a high-quality set of wind speed and direction data at the Jornada Experimental Range in summer 2014, using the same logger technology as the Lorenz and Jackson (2015) effort. These data, uncontaminated by vehicle motion effects, allowed rather accurate vortex model fits, e.g. Fig. 11, wherein the pressure, wind speed and direction histories are simultaneously fit. The superposition of the circumferential vortex winds and the ambient wind field result in quite distinctive wind direction and speed histories, which resolve most of the geometric ambiguities intrinsic to fitting a pressure time series alone.

\subsubsection{Other Terrestrial Dust Devil Observations}

Electric Fields/Saltation-Dust Flux Dust on Earth is mainly lifted from the surface through the process of 'saltation' (Bagnold 1941; Shao 2008): when wind friction velocity/surface stress overcomes a threshold, its drag force causes larger particles with size around 100 micrometers to be the first to move. They jump over the surface, where they reimpact and initiate the motion of particles of a wide range of sizes, including dust. Indeed, due to dust sized particles protruding minimally upward into the wind affected near-surface atmosphere and also strong interparticle forces, dust grains are difficult to be lifted directly by the wind force (Gillette et al. 1974; Greeley and Iversen 1985; Shao et al. 1993).

The collisions among particles during saltation are also responsible for electric charge transfer between grains. Even if the exact mechanism for this process is still not clearly understood, some experiments and observations show that this process is size dependent (Freier 1960; Inculet et al. 2006; Duff and Lacks 2008; Lowell and Truscott 1986; Kok and Renno 2008; Desch and Cuzzi 2000; Forward et al. 2009; Gill 1948; Latham 1964; Latham and Stow 1968; Harper 1967; Horn et al. 1993; Lacks and Levandovsky 2007). Considering that, in general, the smallest particles are transported higher into the atmosphere by local turbulence while larger particles remain closer to the surface, this translates in a charge separation and consequently in an enhancement of the atmospheric electric field.

So generally, a variation of the electrical properties of the atmosphere is observed during dust events including dust storm and dust devils with electric fields up to $150 \mathrm{kV} / \mathrm{m}$ being measured (Esposito et al. 2016; Schmidt et al. 1998; Renno et al. 2004; Kok and Renno 2006, 2008; Harper 1967). Fig. 12 shows an example of electric field observed during a dust devil (Esposito et al. 2016).

Esposito et al. (2016) undertook field test campaigns in the West Sahara desert to study dust lifting process by monitoring simultaneously weather parameters (pressure, wind, relative humidity, temperature, solar irradiance), soil properties (temperature, moisture), sand and dust dynamics (dust size distribution and abundance, sand saltation rate and flux), and the atmospheric electric field (with a field mill). They monitored several dust storms and devils. They found that there is a very strong correlation between the concentration of dust lifted during a dust storm and the atmospheric electric field intensity. The same behavior was observed also during dust devils, indicating that a similar dust electrification process was in action (Fig. 13). 

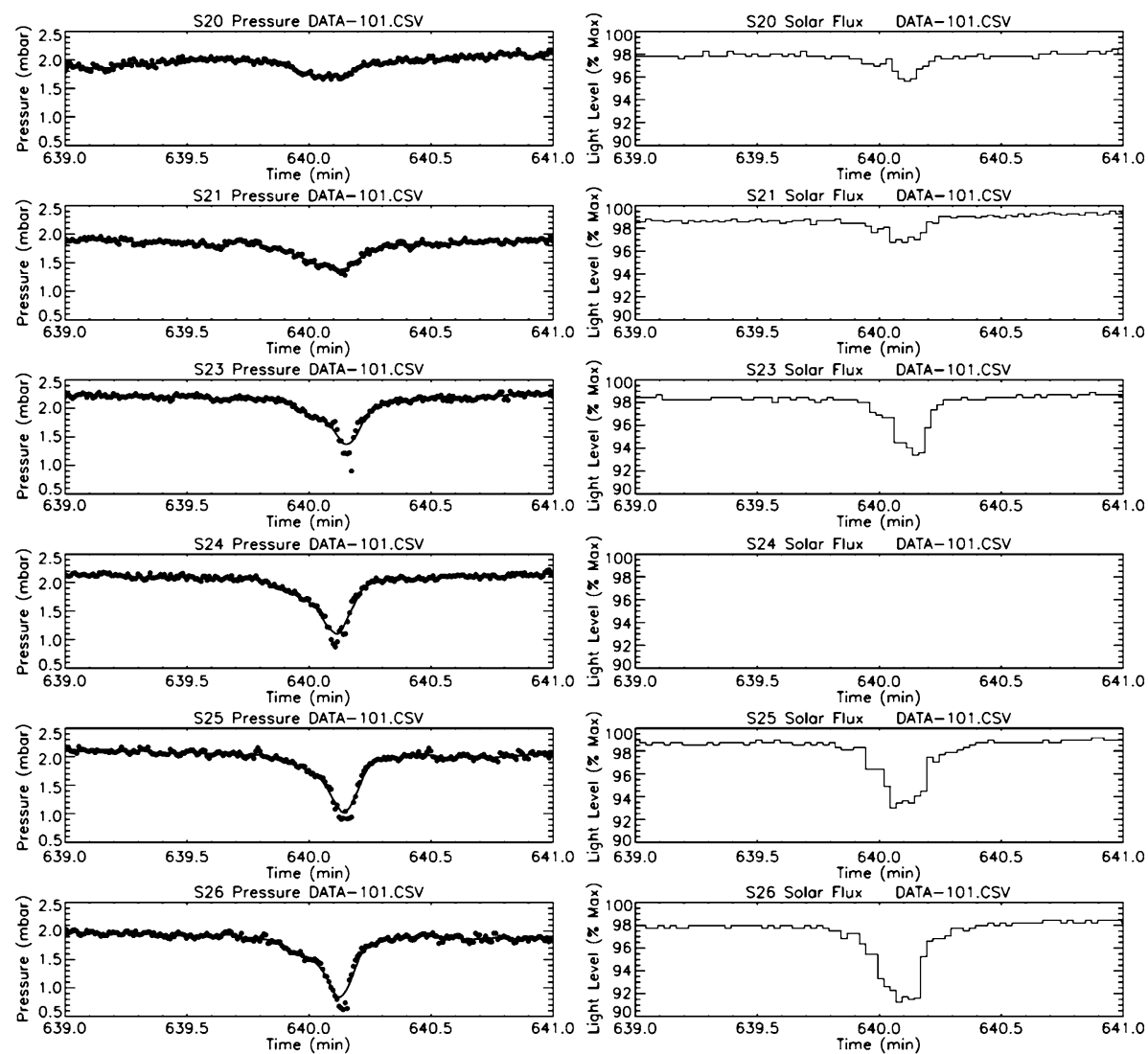

S27 Pressure DATA-101.CSV
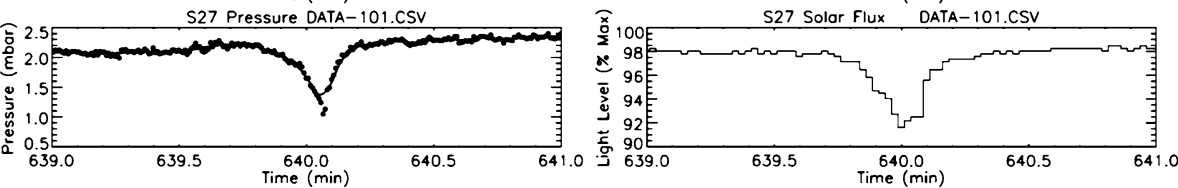

S28 Pressure DATA-101.CSV
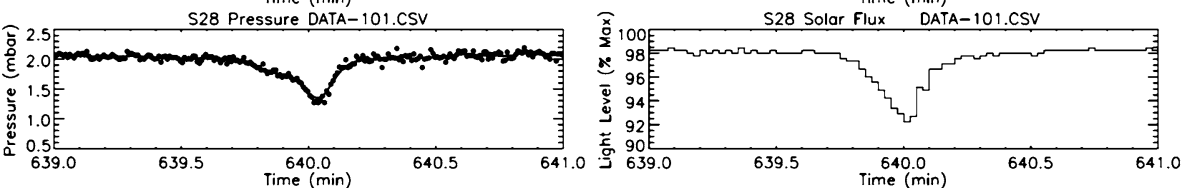

S29 Pressure DATA-101.CSV
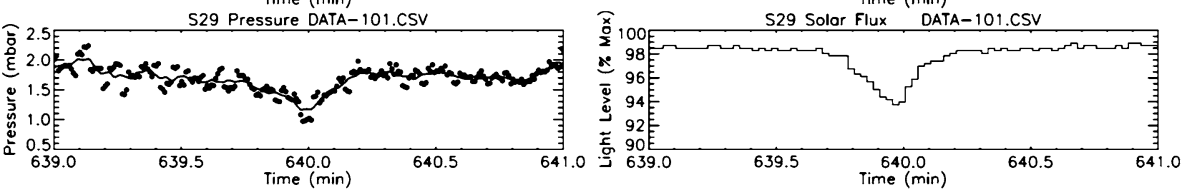

Fig. 10 A 'bullseye' encounter at La Jornada, where a dust devil swept across a 10-station pressure/solar logger array. A signature is visible in all stations, but falls off in amplitude to either side. The plots are a 2-minute record from each station, with pressure (normalized to the beginning of the original datafile) plotted on the left, and solar flux (normalized to maximum) on the right. The devil is dust-laden, as evidenced by the sunlight drop that is simultaneous with the pressure signal. Note that no solar data is available on station S24 (which had been flipped over, perhaps by animal action). Note that the profile is asymmetric-the onset of the pressure drop is shallower and longer than the decay, a feature often seen and perhaps associated with the advection of the devil in the ambient wind field. [After Lorenz et al. 2015b] 

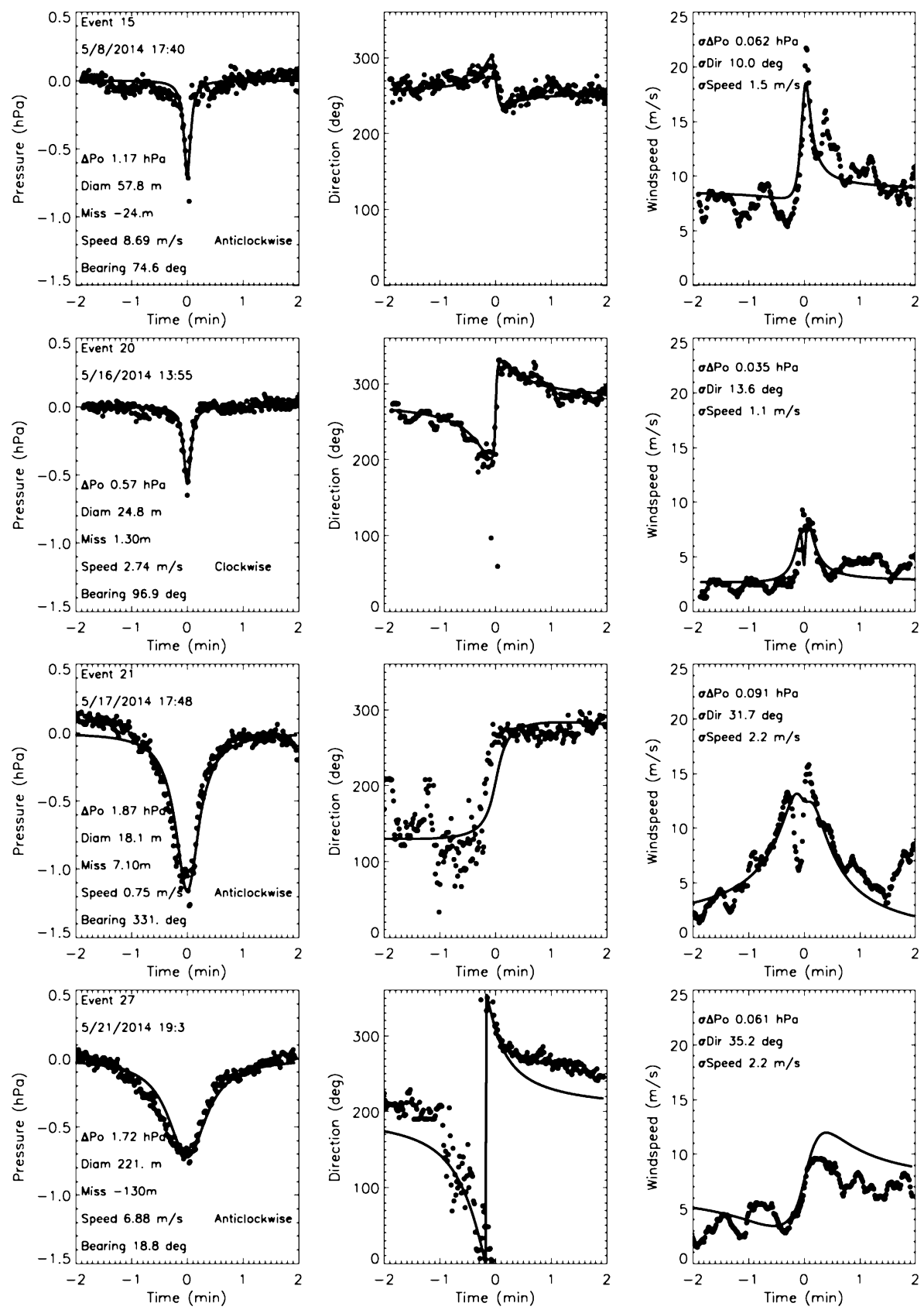

Fig. 11 Three encounters with 1-Hz pressure, wind direction and speed (left to right) data (grey points) of dust devils at La Jornada (Lorenz 2016) with vortex model fits (black lines). The wind direction histories are particularly distinctive-speed histories tend to be somewhat noisy. It may be noted that the 'eye' of the vortex, where near-solid-body rotation within the wall results in very reduced windspeed at the center of the devil, clearly resolved in the lower-right plot (although not well captured by the model fit) 
Fig. 12 An example of the electric field accompanying a dust devil as measured by Esposito et al. (2016) in the West Sahara desert: atmospheric electric field enhancement

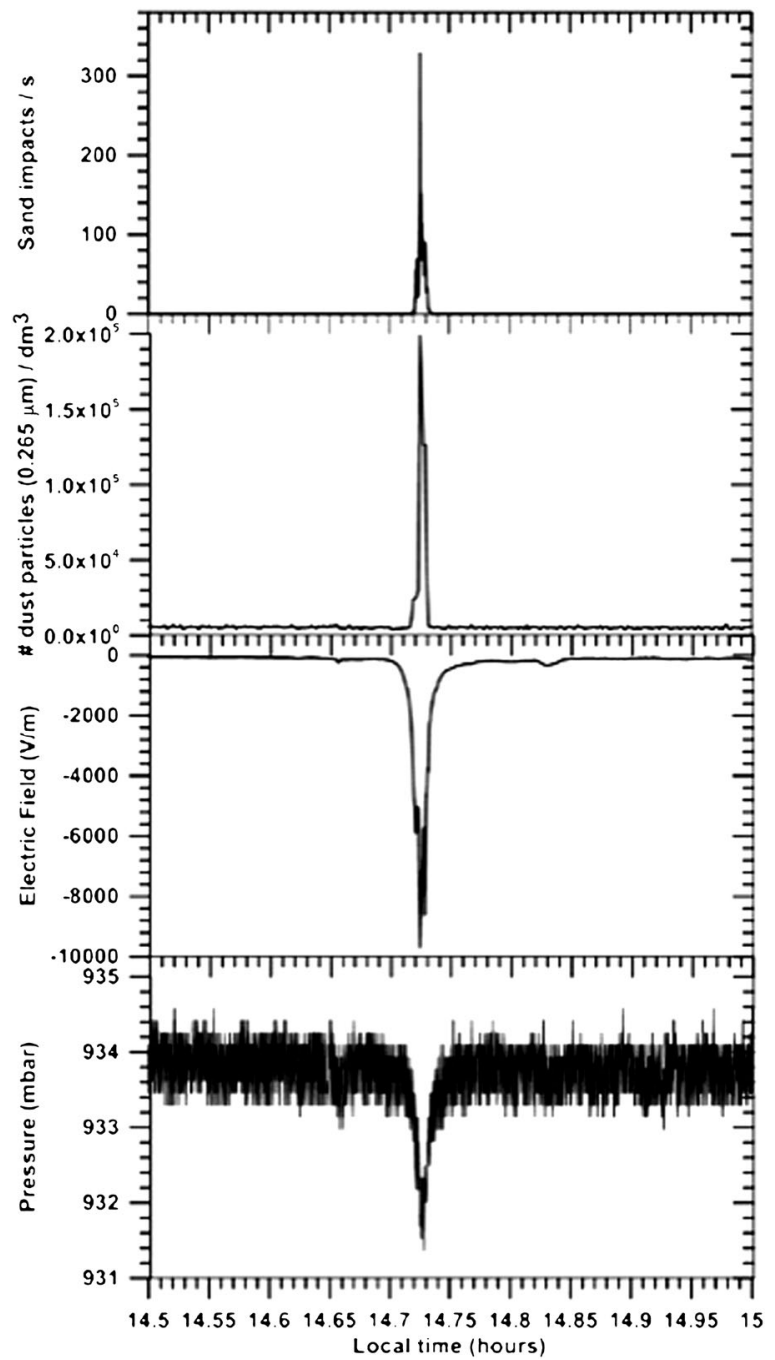

The 2014 West Sahara Campaign depicted in Esposito et al. (2016) has also been the opportunity to test the Micro-ARES electric field sensor of the DREAMS (Dust Characterisation, Risk Assessment, and Environment Analyser on the Martian Surface) science package for ExoMars 2016 (Bettanini et al. 2014). The instrument is based on the Relaxation Probe principle (Berthelier 2000, Molina-Cuberos et al. 2010) which requires a more complex post-processing than a classic field-mill sensor. One of the capabilities that had to be tested was the ability for the instrument to detect the electric field variations during the nearby passage of a dust devil, the passage being confirmed by the classical weather measurements. It appears that such events have been observed (Fig. 14) during the 4 days campaign and properly detected by the instrument. A more detailed study of the results will show if the instrument is able to detect single particle collisions with the electrode, thus giving access to the particle electric charge during such events. A more detailed overview of the 
Fig. 13 Correlation between the abundance of lifted dust and the intensity of atmospheric electric field during dust storms (red marks) and dust devils (blue marks)
Fig. 14 Comparison of Micro-ARES and Commercial Field-mill sensor DC measurements during a dust-devil event confirmed by pressure and wind parameters measurements. The amplitude and time differences are explained by the installation height (respectively 0.8 and $2 \mathrm{~m}$ ) of the instruments and their distance (approx. 30 meters)

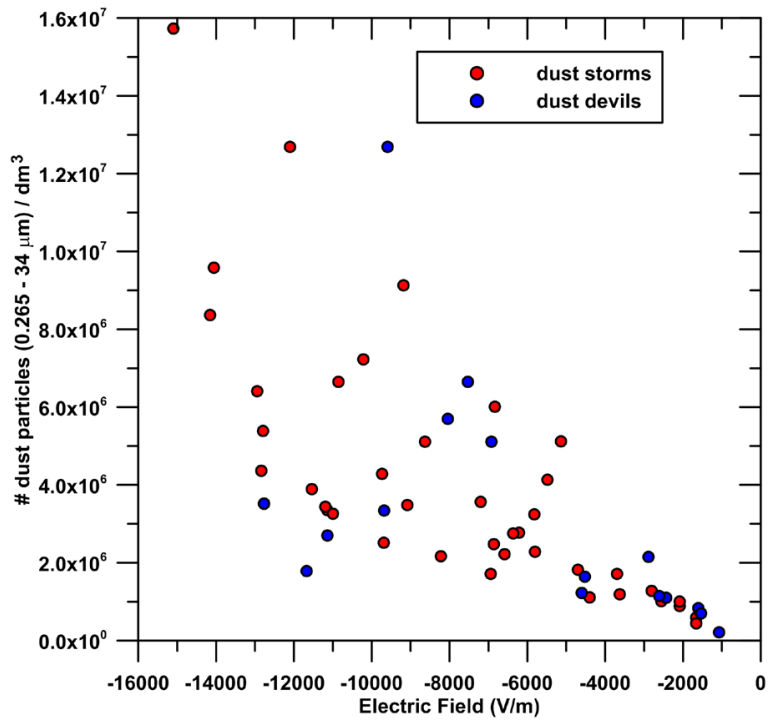

11 July 2014

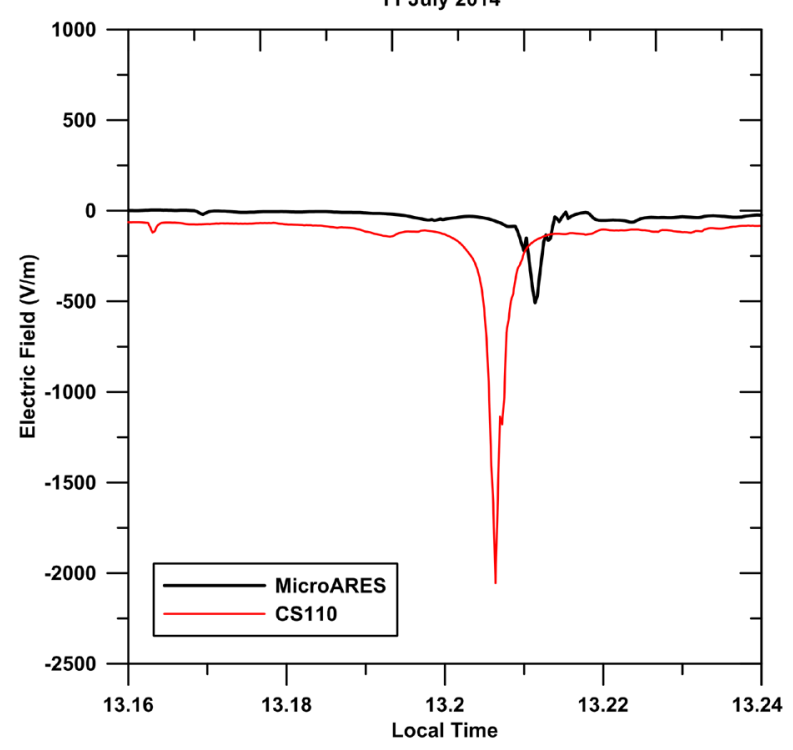

Micro-ARES and the results gathered during the 2014 West Sahara Campaign is presented in Harrison et al. (2016) in this issue.

Seismic Signals Pressure fluctuations in the atmosphere induce an elastic response in the ground that can be detected as a ground tilt by seismic stations installed on, or close to, the Earth's surface. This effect has been known since the 1970s (Sorrells 1971; Sorrells et al. 1971) and is one of the reasons that terrestrial seismic stations are typically installed deep underground in vaults. 
Fig. 15 Two distinct seismic signals recorded during the passage of two dust devils during mid-afternoon in late spring (2014) on a playa in the Mojave Desert, USA. The seismic measurements were accompanied by pressure and wind measurements; time was not precisely synchronized between the two sets of measurements; the meteorology measurements have been time shifted to coincide with the seismic signals. A third seismic signal (at $\sim 19$ minutes) is not accompanied by a meteorologically detected dust devil. [After Lorenz et al. 2015b]
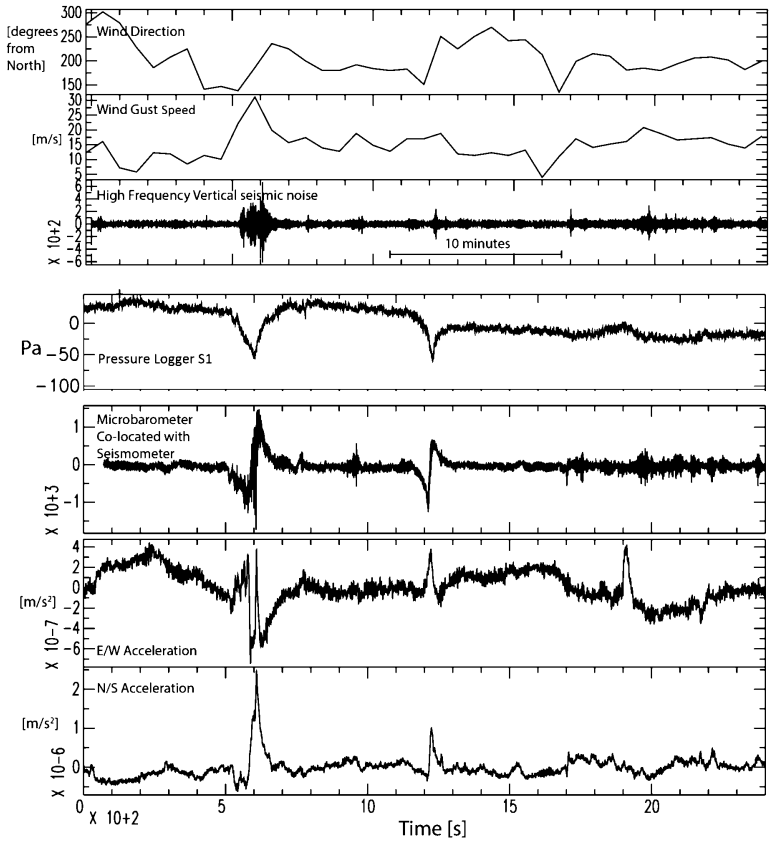

The InSight lander mission, selected under the NASA Discovery programme and now scheduled for a May 2018 launch, will perform the first comprehensive surface-based geophysical investigation of Mars. The seismic instrument SEIS (Seismic Experiment for Internal Structures) is the critical instrument for delineating the deep interior structure of Mars (Lognonné et al. 2012). SEIS will be deployed directly onto the surface of the planet and will, therefore, be sensitive to the atmospheric seismic signals. In fact, due to the lack of microseism-producing oceans, such atmospheric seismic signals are likely to be the dominating background seismic noise on Mars (Lognonné and Mosser 1993).

In preparation for the InSight mission, and to understand the effects of a surface deployment of a seismometer, representative field experiments were carried out in California close to the Goldstone Deep Space Communications Complex (Lorenz et al. 2015a). The experiment included a seismometer buried at very shallow depth, together with a suite of meteorological instruments. During this field campaign, ground tilt was measured by the seismometer at the same time as vortex encounters were documented by an array of pressure loggers (Lorenz et al. 2015a). The negative load of a dust devil vortex pulls up the ground as it passes, causing the ground - and seismometer - to tilt away from the dust devil center. This first identification of the isolated seismic signature of a dust devil has shown that a seismometer appears to be capable of tracking close encounters with dust devils and, in addition, that seismometers may be more effective than in-situ meteorological instruments at detecting dust devils at long-range (Lorenz et al. 2015a).

Peak surface accelerations of $1-2 \times 10^{-6} \mathrm{~m}^{2} \mathrm{~s}^{-1}$ were measured during the passage of a dust devil presenting a measured pressure drop of $80 \mathrm{~Pa}(0.8$ millibars $)$ accompanied by a measured $2 \%$ decrease in the short-circuit current of a solar cell mounted with a pressure logger located 30 meters from the seismometer (Fig. 15). The measured acceleration magnitudes and knowledge of the local surface material structure provided by a seismic survey enabled estimation of the dust devil's decreased surface mass loading/negative point load on an elastic half-space. The measured acceleration values and inferred vortex center miss 
Fig. 16 Microbaragraph measurement of a dust devil pressure signature, showing the up-down heartbeat signature of the vortex. Two additional smaller amplitude subsidiary events are present $\sim 1$ minute before and 2 minutes after the primary event. [After Lorenz and Christie 2015]

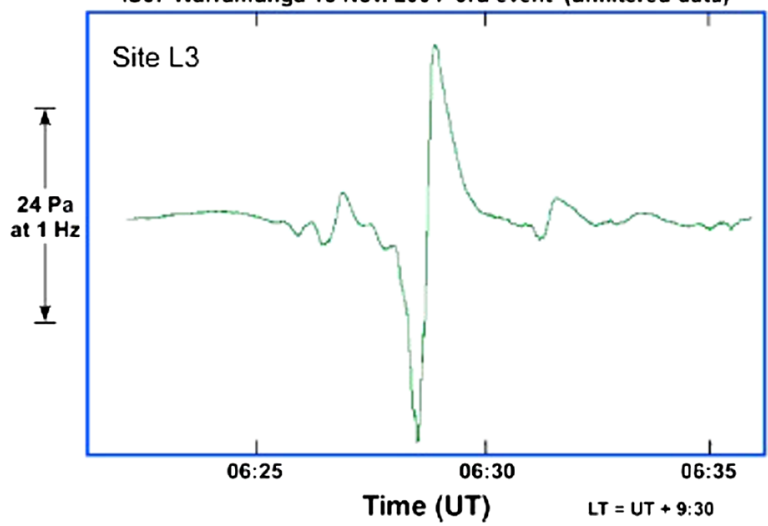

distances imply a mass load of $\sim 8000$ Newtons and tilt accelerations of $7 \times 10^{-7} \mathrm{~m} \mathrm{~s}^{-2}$ for a 5-meter diameter vortex with a central pressure deficit of $200 \mathrm{~Pa}$ passing 10 meters from the seismometer. A larger diameter (100 meter) dust devil also with a $200 \mathrm{~Pa}$ central pressure deficit could provide a total mass load of 300 metric tons and accelerations of $10^{-6}-10^{-5} \mathrm{~m} \mathrm{~s}^{-2}$ for miss distances of 50-200 meters, though the size of such a dust devil violates consideration of it as a point-source. The seismometer system employed by Lorenz et al. (2015a, 2015b, 2015c) included a microbaragraph that coincident with the seismic signals registered the tell-tale infrasound 'heartbeat' dust devil signature identified by Lorenz and Christie (2015).

Infrasound and Acoustic Measurements It was noted by Lorenz (2012a, 2012b) that while meteorological stations tend to record data at only 15 -minute intervals, continuous pressure measurements, made with sufficient sensitivity and sample rate to detect dust devil vortices are made for other applications, notably for monitoring compliance with international treaties on nuclear testing. Some of these stations, operated by the Comprehensive Test Ban Treaty Organization (CTBTO) are located in desert areas, and dust devil vortex signatures at a CTBTO station in Australia are reported by Lorenz and Christie (2015). Since atmospheric effects are a major perturbation to seismic signals, many seismic stations (such as those in the USARRAY) now also record pressure and other meteorological parameters. Data-mining of such records may be a fruitful avenue of research. A subtlety to be noted is that microbarographs used for infrasound studies (and those e.g. at CTBTO stations) are high-pass-filtered pressure records, such that the principal component in the signal is the derivative of the pressure signal. Thus the typical dip seen in pressure time series in fact appears as a down-up-down 'heartbeat' signature (Fig. 16).

Lorenz and Christie (2015) investigated dust devil pressure signatures within microbaragraph measurements obtained as part of CTBT monitoring from a station located in Australia. The high pass filtered signal of a dust devil pressure measurement appears as a 'heartbeat' signal that resembles the temporal derivative of measured pressure provide by a pressure sensor with a sampling of $\sim 1 \mathrm{~Hz}$. This heartbeat signal exhibits declining signal magnitude as the pressure minimum is approached and an abrupt transition in the sign of the signal to a maximum magnitude that subsequently declines in concert with the measured pressure increase as the dust devil's influence wanes. Contemporaneous microbaragraph and pressure sensor measurements verify the dust devil production of the microbaragraph's 
heartbeat signal. While this is not a direct measure of the infrasound generation produced by a dust devil, it is a distinct signal from which dust devil occurrence can be derived.

Edmonds (2014) attempted to detect the infrasound emission that a dust devil, or that interacting dust devil vortices, might produce. Theory (Powell 1964; Howe 2003) indicates that two vortices 'orbiting' around each other are capable of generating infrasound emission, as is a single non-circular vortex (Howe 2003). Williams (2001) addressed the attenuation of acoustic signals within Mars' tenuous atmosphere and concluded that infrasound frequencies $(<20 \mathrm{~Hz})$ at which dust devils are effective emitters (Bedard 2005) experience smaller dissipation than do higher audible frequencies. That work was motivated by the inclusion on the ill-fated 1999 US/NASA Mars Polar Lander of a microphone intended to listen for martian sounds. Edmonds' (2014) work was motivated by the inclusion on the NASA InSight lander of a high-frequency pressure sensor (Banfield 2014). Edmonds (2014) conducted a field exercise in the desert of southern New Mexico, USA in Spring 2014 attempting to detect the infrasound and audible frequency emission from dust devils. Using a microphone/recording acoustic system and a microbaragraph, three dust devils recorded from distances of a few to 10's of meters coincided with measured audible $(>20 \mathrm{~Hz}$ ) signals. The recordings indicate amplitude 'ridges' within distinct acoustic frequency ranges, distinct from sounds attributed to wind-induced movement of vegetation objects (vegetation, sand, etc.). However, no infrasound detection was identified.

\subsection{Mars Surface Obtained Meteorological Measurements of Dust Devils}

While there has been until recently a general dearth of seasonal or longer temporal coverage of terrestrial dust devil in situ measurements arising from limited duration measurement campaigns, but a plethora of measured dust devil parameters/characteristics from these same studies, in situ measurement of martian dust devils has suffered from opposite conditions. Martian lander and rover missions have provided durations extending from 83 sols (Pathfinder) to multiple martian years (Viking Lander 1 spanned more than 3 Mars years, Spirit Rover 3 Mars years, Opportunity Rover 6+ Mars years and continuing, and MSL almost two Mars years and counting). However, some missions have suffered from meteorological instrument failures (Viking Lander 1, MSL) or calibration issues (Pathfinder) while others did not carry any direct meteorological instruments at all (Opportunity, Spirit). The robotic vehicles that did carry meteorological instrumentation generally have provided high frequency, one to a few $\mathrm{Hz}$, sampling sufficient to characterize dust devil signatures but did not do so continuously, except for the Phoenix Lander. It is from the measurements provided by the five meteorology-instrumented vehicles (Viking Landers 1 \& 2, Pathfinder, Phoenix, MSL) that our current thermodynamic understanding of martian dust devils has been obtained.

A compilation of measured characteristics is provided in Table 2.

\subsubsection{Viking Landers}

The two US/NASA Viking Lander spacecraft which arrived at Mars in 1976 provided the first opportunity for in situ sensing of the meteorological signatures of passing martian dust devils. Viking Lander 1's landing location was 23 N, 48 W, while Viking Lander 2's was $48 \mathrm{~N}, 226 \mathrm{~W}$.

As previously described, a characteristic transient drop in atmospheric pressure is detected when a convective vortex passes over/near a deployed pressure sensor, as has been amply demonstrated for terrestrial dust devils. The digital quantization of the long lived 
Viking lander pressure sensors was $8.8 \mathrm{~Pa}$ (Hess et al. 1977; Tillman et al. 1993), which subsequent missions (Murphy and Nelli 2002; Ellehoj et al. 2010) indicated was too large to unambiguously detect a martian dust devil pressure signature of several Pascals or less magnitude. Additionally, the Viking Lander pressure measuring strategy was not generally focused upon detection of short duration events but rather upon characterization of diurnal and seasonal variations. A typical time interval between pressure measurements was $17 \mathrm{~min}-$ utes (Ryan and Lucich 1983). Pressure sampling did include some brief time periods early in the mission during which measurements were obtained at a rate of $\sim 1$ per second, but much more often sampling rates were once each 16 or 32 seconds extending to once per 65 to 105 minutes. There is no publication that addresses assessment of the complete Viking lander pressure record for identification of dust devil/convective vortex signatures. The VL1 pressure record spans from $L_{s} 97.1$ (MY 12) through $L_{s} 226.7$ (MY 15), while VL2 spans $L_{s} 117$ (MY12) through $L_{s} 57.1$ (MY 14).

While Viking Lander pressure measurements were not amenable to dust devil studies, Viking's measured winds were. Ryan and Lucich (1983) investigated wind vector measurements provided by Viking Meteorology Instrument System (VMIS) at both landers, with measurement sampling intervals ranging from 2-112 seconds. Vortices were identified by temporal rotation of the measured wind direction accompanied by a concurrent wind speed variation illustrative of an imbedded Rankine-type vortex, and a concurrent temperature maximum. A total of 118 vortices were identified during the mission's first year, 40 vortices at VL1 spanning summer through winter and 78 at VL2 spanning summer through spring. The greatest likelihood of vortex detection occurred almost equally $(\sim 65 \%$ of the sols investigated) at VL1 during summer and VL2 during spring.

Identified vortex disturbance influence persisted for several to $\sim 10$ minutes, with the most pronounced vortex effects present for tens of seconds to several minutes. Inferred vortex core diameters, the distance from vortex center at which tangential wind speed maximized, were generally tens to several hundreds of meters, with radii of disturbance effects extending out ten times the core radius. Several inferred core diameters extended to $\sim 500-1000$ meters, implying radii of vortex influence extending to $\sim 5-10 \mathrm{~km}$. Inferred vortex rotation was equally divided between cyclonic ('counter-clockwise') and anticyclonic ('clockwise'), consistent with terrestrial experience (Sinclair 1973) and suggestive that the vorticity is generated locally at small scales. Several of the vortices at both Viking lander sites were sufficiently intense to generate winds $\left(>35 \mathrm{~m} \mathrm{~s}^{-1}\right)$ deemed capable of lifting surface dust (Greeley and Iversen 1985). There were no concurrent imaging observations invoked to address the presence or lack of dust in the detected vortices.

It is unclear how unambiguous VL1 wind directions employed in this investigation were derived subsequent to sol 45 and the failure of the wind direction quadrant sensor (Murphy et al. 1990). VL2's wind instrument did not suffer from such a failure.

Ringrose et al. (2003) readdressed the dust devil/convective vortex signatures present in Viking Lander 2 wind measurements. Using a phase picker detection technique comparing a running mean value to a threshold value (also used terrestrially in Hecht et al. 2001), instances where a short term average wind speed or wind direction varied by more than $6 \mathrm{~m} \mathrm{~s}^{-1}$ or 40 degrees azimuth, respectively, from longer term averages were flagged as possible vortex signatures. For verified vortex signatures, minimum distance from the vortex center and vortex diameter were derived from a Rankine vortex fit to the measured wind. Applying this technique to VL2's first 60 sols resulted in 38 identified vortex occurrences, nine of which suffered from lander interference which makes them suspect. Maximum measured wind speeds were $12-15 \mathrm{~m} \mathrm{~s}^{-1}$ and maximum wind vector rotation was 300 degrees. Inferred vortex core diameters arising from the Rankine vortex fits ranged from a few 10's to 
a few hundred meters. Detected vortex durations ranged from 60-1080 seconds. Maximum inferred vortex core diameter tangential wind speeds were $\sim 100 \mathrm{~m} \mathrm{~s}^{-1}$ for two events. These two events coincided with the largest inferred 'miss distance from core center' $(\sim 1700 \mathrm{~m})$ and largest vortex core diameters $(>350 \mathrm{~m})$, while maximum inferred speeds for smaller $(<250 \mathrm{~m})$ miss distances and smaller core diameters $(<100 \mathrm{~m})$ ranged from 3 to $70 \mathrm{~m} \mathrm{~s}^{-1}$. Daytime hour-of-occurrence of detected vortices exhibited late morning and early afternoon maxima. A secondary minimum was also evident at 0930 local time. There was no description of the completeness of coverage of measurements during the 30-minute time intervals into which the vortex occurrences were binned for this time-of-sol evaluation, so the timeof-sol distribution might not be representative of conditions.

Measured atmospheric temperature increases of several degrees Centigrade accompanied some, but not all, of the wind-detected vortices identified by both Ryan and Lucich (1983) and Ringrose et al. (2003).

The Viking Landers did provide for the first time in situ evidence of martian convective vortices, in the form of wind vector temporal variation consistent with the passage of a vortex. Vortex occurrences were frequent, with at least one-half of the mission sols investigated possessing a detected vortex occurrence. This detection rate is certainly a lower limit since VMIS was not in continuous operation during any of the sols investigated.

\subsubsection{Mars Pathfinder}

From its northern subtropical landing location, Pathfinder provided pressure measurements that spanned the mission's 83 late summer-early autumn sols. Pathfinder's deflecting diaphragm, variable reluctance pressure sensor (Seiff et al. 1997) provided 14-bit, $\sim 0.25 \mathrm{~Pa}$ resolution in its 600-1000 Pa surface operating mode. While Pathfinder's Atmospheric Structure Investigation/Meteorology (ASI/MET) system (pressure, temperature, wind) generally provided greater temporal sampling resolution $(0.25$ to $2 \mathrm{~Hz})$ than did the Viking Lander's, ASI/MET like Viking VMIS was not continuously operated. During the mission's first $\sim 30$ sols, 3-minute, $0.25 \mathrm{~Hz}$ measuring sessions initiated at the start of most LTST hours were augmented by 15 -minute and 60 -minute continuous measurement sessions at $1 \mathrm{~Hz}$ sampling cadence. On five occasions during the mission, the first starting at 0600 LTST of Sol 25, the ASI/MET system was continuously operated for a complete sol at a sampling rate of $0.25 \mathrm{~Hz}$. These 'Presidential MET' sessions were initiated on Sols 25, 32, 38, 55, and 68. Subsequent to Sol 30, the ASI/MET system was operated during daylight hours only, 0900-1600 LTST, except during the Presidential MET sessions.

Schofield et al. (1997) presented the first in situ contemporaneous measurement of pressure, wind and temperature within a martian dust devil/convective vortex, from measurements obtained during early afternoon, 1353 LST, during the Sol 25-initiated Presidential MET session (Fig. 17). The quantified vortex pressure drop magnitude was $\sim 2.5 \mathrm{~Pa}$. Subsequently, Murphy and Nelli (2002) assessed the entire Pathfinder pressure data archive and identified the occurrence of 79 vortices, $\sim 1$ per sol, with pressure drop magnitudes equal to or exceeding 0.5 Pa. Vortex identification was based upon a pressure drop magnitude determined from the difference between a 3rd order polynomial fit to measured pressures during a 15-minute interval and the unaltered pressure measurements. The maximum pressure drop magnitude identified was 4.8 Pa at 1132 LST on Sol 34. The LST time of detection of the 79 vortices spanned $0930-1700$.

The discontinuous temporal coverage provided by ASI/MET indicated that the true number of detectable vortices would exceed 79. Normalizing the number of vortices detected throughout the mission during each 15-minute LST time window by the percentage of time 
Fig. 17 Mars Pathfinder ASI/MET measured Time series of $0.25 \mathrm{~Hz}$ pressure, temperature, and wind direction during a 4-minute time period during the early afternoon of Sol 25 of the mission. After Schofield et al. (1997)

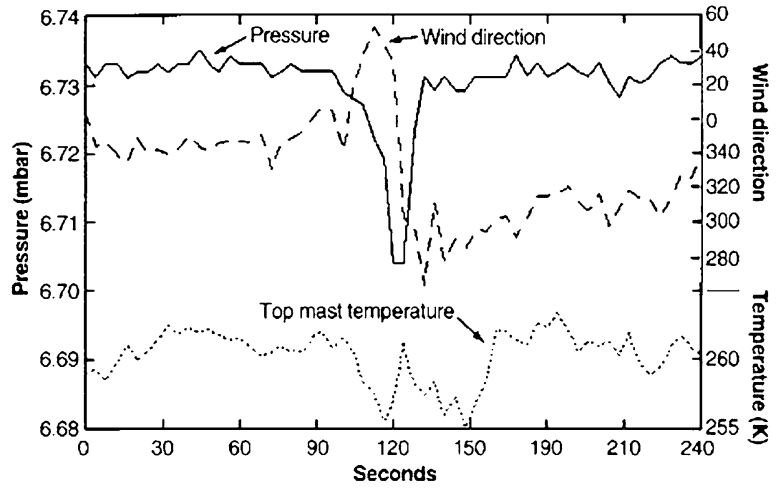

the ASI/MET system was operating during that 15-minute window resulted in an estimate of 210 detectable vortices occurring during the 83 sol mission, equating to $\sim 2.5$ vortices per sol. This 2.5 per sol estimate is less than the $\sim 4$ vortices per sol detected during the five Presidential MET sols of almost continuous pressure sensor operation (Murphy and Nelli 2002).

Ferri et al. (2003) determined the duration of 19 of the larger magnitude ( $>1 \mathrm{~Pa}$ ) pressure signature events identified by Murphy and Nelli (2002). Durations ranged from 14-51 seconds, with a mean value of 28 seconds and median value of 25 seconds.

Both the directly detected and normalized inferred Pathfinder vortex occurrences exhibited a maximum hourly occurrence during very early afternoon (Fig. 18). Vortex activity after the early afternoon peak declined more gradually than through the afternoon than its increase during the morning.

Applying Metzger et al.'s (1999) visible plume dust mass load estimates and the frequency of occurrence derived from the Pathfinder pressure measurements, Murphy and Nelli (2002) estimated that local dust devil activity could provide dust to the atmosphere at the rate of an optical depth of 0.01 per sol over a $1.5 \mathrm{~km}$ diameter area centered upon the lander. This estimate is approximately equal to the opacity decay rate measured during the decline of the second global scale martian dust storm during 1977 (Pollack et al. 1979).

Pathfinder's ASI/MET system included a wind sensor (Seiff et al. 1997). Additionally, a 'wind sock' was mounted on the ASI/MET mast with IMP-provided images enabling wind vector derivation from observed windsock orientation (Sullivan et al. 2000). The ASI/MET wind sensor was a thermal/mechanical instrument using the measured overheat of resistively heated sensor elements (wires) from which wind speed and direction were intended to be derived. The sensor consisted of a vertically oriented $2.7 \mathrm{~cm}$ diameter, $3 \mathrm{~cm}$ tall solid exterior cylinder around which were arrayed six 'segments' of azimuthally confined vertically aligned 8-wire length windings. A pulsed 20 milliamp current provided unheated segment wire temperature from measured voltage drops across each segment. When the sensor was operated in a continuous 52 milliamp high current mode the segment wire temperatures were resistively heated. Segments positioned in the upwind direction experienced ventilative cooling, with overheat magnitudes and their azimuthal structure providing the signal indicative of the wind vector. The sensor was not designed with its own reference temperature against which segment temperature could be assessed for 'overheat' magnitude. It was anticipated that thermocouples mounted on the ASI/MET would provide the reference temperature. However, large magnitude 'turbulent' temperature variations $(\sim 10-15 \mathrm{~K}$; Schofield et al. 1997) inhibited unambiguous determination of a reference temperature for wind sensor segment overheat determination purposes. 
Fig. 18 Martian dust devil occurrence frequency versus time-of-sol from pressure signatures from Mars Pathfinder (top; Murphy and Nelli 2002), Mars Phoenix (middle; Ellehoj et al. 2010) and Mars Science Lab (bottom; Steakley and Murphy 2016)
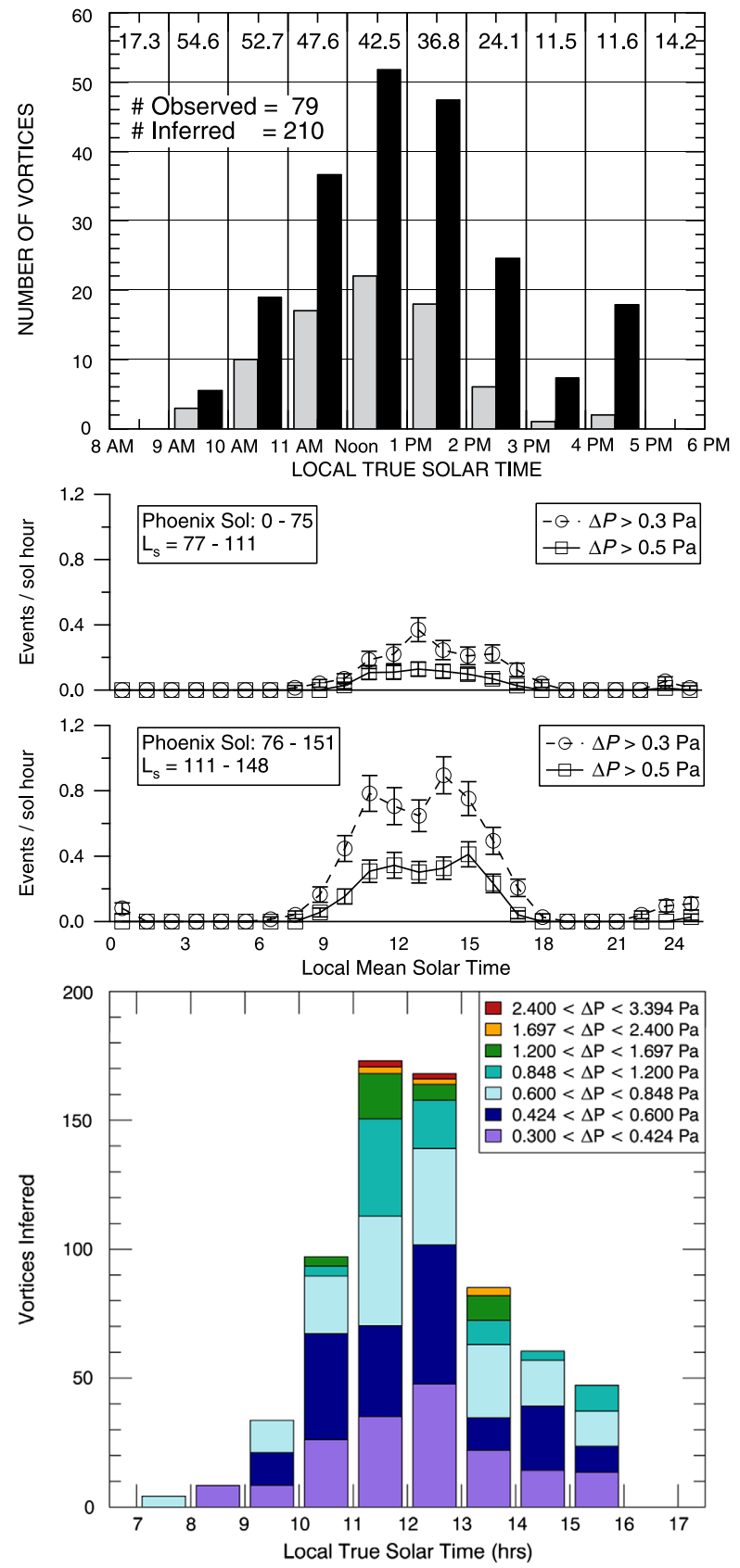

The wind sensor was generally operated for $\sim 12$ seconds of low-current pulsed mode to establish unheated segment temperatures, after which continuous high current was imposed for $\sim 150$ seconds. It would be during these 150 second intervals that wind speed and direction could be quantified. 
Despite these unresolved operational/calibration issues for the Pathfinder wind sensor, the sensor did provide signals that were qualitatively correlated to dust devil occurrences identified in Pathfinder's pressure measurements (Murphy and Nelli 2002; Schofield et al. 1997). Wind sensor signals did suggest substantial and abrupt changes in wind direction and speed in conjunction with measured dust devil pressure signatures (see, for instance, Fig. 8 in Schofield et al. 1997), however no systematic study of vortex winds has been published.

In addition to the ASI/MET's thermal wind sensor, Pathfinder also included three mechanical 'wind socks' mounted at three heights along the ASI/MET mast (Sullivan et al. 2000). IMP provided images of the solid, metallic, inverted-cone shaped socks and their tilt orientation from which wind speed and direction were derived using pre-flight calibration information. Twelve image sequences obtained over a time period of $\sim 100$ seconds several times per sol provided the dataset from which wind vector derivation was attempted. Data from only four of the sampled 42 sols provided imaging indicative of minimum wind speed (few meters per second) necessary to overcome windsock inertia and induce detectable windsock deflection (Sullivan et al. 2000). Thus, Pathfinder's windsock experiment did not aid in advancing understanding of the dynamic components of martian dust devils.

Schofield et al. (1997) noted that one pressure-indicated vortex passage detected by MPF was accompanied by a transient drop in output from the lander's solar panels, suggesting that the vortex was dust-laden and the dust column created at least a partial shadow that crossed the lander. This observation underscores the utility of making available engineering data such as solar array current data from lander missions-in effect the arrays serve as a 'free' instrument.

\subsubsection{Mars Exploration Rovers (Spirit and Opportunity)}

Neither Spirit nor Opportunity were outfitted with meteorological measuring instrumentation. The MERs' mini-TES instrument was used to diagnose the vertical atmospheric structure within the bottom few hundred meters to 1-2 km (Smith et al. 2004). While those measurements illustrated the superadiabatic conditions present during mid-sol, there was no identification of such a measurement probing a dust devil.

It had been noted during the MPF mission that a calibrated solar cell on the Sojourner rover recorded a progressive decline in cell current of $\sim 0.25 \%$ per day (Landis and Jenkins 2000), due to the accumulation of airfall dust on the cell. This obscuration set expectations of operational lifetime of solar-powered landers and rovers on Mars (e.g. the Mars Exploration Rovers Spirit and Opportunity had a nominal mission duration of 90 days).

In practice, it was observed that while the MER solar power per day declined due to dust accumulation, sudden reversals of the decline were seen, and camera images of the arrays showed that dust had been removed. While dust devils were suspected, the likelihood remains that straight-line gusts may have been responsible. However, Lorenz and Reiss (2015) showed that not only did the seasonal onset of dust-clearing events coincide with the appearance of dust devils (Greeley et al. 2006; 2010), but also the rate at which dust-clearings occurred was coincident with the rate of vortex encounters seen in pressure drops by Phoenix and Pathfinder, extrapolated to a dust lifting threshold of a few Pa.

\subsubsection{Mars Phoenix Lander}

The US/NASA Phoenix lander meteorologically detected, at its northern arctic latitude landing site, dust devil/convective vortex signatures that complement its SSI imaging dust devil 
detections (Sect. 2.2.3). Phoenix was equipped with a meteorological package (MET), including pressure, air temperature and wind sensors and a Light Detection And Ranging (LIDAR) instrument for measuring dust and ice particles in the atmosphere (Taylor et al. 2008; Whiteway et al. 2008). The pressure sensor was based on Barocap ${ }^{\circledR}$ silicon diaphragm sensor heads manufactured by Vaisala Inc. and had a very high resolution of $0.1 \mathrm{~Pa}$ (Taylor et al. 2010), limited by the noise level. In contrast to the Vikings, Pathfinder and MSL, Phoenix logged atmospheric pressure and air temperature almost continuously, with an invariant sampling rate $(0.5 \mathrm{~Hz})$ (Taylor et al. 2010; Davy et al. 2010). The mission spanned 151 sols, almost twice the duration of the Pathfinder mission, extending from early spring through mid northern hemisphere summer $\left(L_{s} 77^{\circ}\right.$ to $\left.L_{s} 148^{\circ}\right)$. The continuous Phoenix pressure record enabled the detection of a greater number of convective vortices than had Pathfinder.

Ellehoj et al. (2010) surveyed the entirety of pressure measurements provide by Phoenix and identified $502(\sim 3.3$ per sol) transient pressure drops similar to the vortex signatures that Murphy and Nelli (2002) had detected in the Pathfinder data. The magnitudes of these Phoenix pressure drops ranged from $0.3 \mathrm{~Pa}$ (an imposed lower limit) to 3.6 Pa, with 197 occurrences possessing a magnitude greater than the $0.5 \mathrm{~Pa}$ detection threshold used by Murphy and Nelli (2002). Vortex identification was based on the search for $20 \mathrm{~s}$ long time intervals that fulfilled the following two criteria: 1) mean pressure more than $0.1 \mathrm{~Pa}$ lower than mean of the previous and next $20 \mathrm{~s}$ intervals, and 2) minimum pressure more than $0.3 \mathrm{~Pa}$ lower than mean of the previous and next $20 \mathrm{~s}$ intervals.

The Full Width at Half Maximum (FWHM) durations of events with magnitude $>0.5 \mathrm{~Pa}$ ranged from less than $1 \mathrm{~s}$ to circa $35 \mathrm{~s}$, the mean being circa $9 \mathrm{~s}$ (Table 1 in Ellehoj et al. 2010). [Note that there is a typographical error in the caption of Fig. 7 in the Ellehoj et al. (2010); the shown quantity is actually full duration, i.e. $2 \times$ FWHM (H. P. Gunnlaugsson, Aarhus University, personal communication, 2015)]. It is unclear if these durations are comparable to the durations reported by Ferri et al. (2003) for the Pathfinder pressure drops, as Ferri et al. (2003) did not explain how their durations are defined.

Most transient pressure drop events identified in the Phoenix data occurred between 06:00 and 18:00 Local Mean Solar Time (LMST). However, unlike Pathfinder, Phoenix also detected also 29 events between 21:00 and 01:00 LMST (mostly with the Sun low in the arctic sky), interpreted as being caused by turbulence induced by air passing over Heimdal crater, the only major topographic feature in the vicinity of the lander (Ellehoj et al. 2010). The general shape of the diurnal distribution of vortex activity resembled that detected by Pathfinder, but at the Phoenix site the vortex activity stayed high until circa 15:00 in the afternoon while at the Pathfinder sites the activity started to fall already at circa 13:00.

Phoenix operated long enough to detect some seasonal variation in vortex activity. The number of identified transient pressure drops generally increased around Phoenix sol 75 ( $L_{s}=111$, about 40 sols after summer solstice and 15 sols before the Sun set for the first time) and the proportion of events with large pressure drops became higher at the same time (Ellehoj et al. 2010). Before this, an average of 0.6 events with magnitude $>0.5 \mathrm{~Pa}$ were observed per sol, but after this 2.0 events per sol, a number comparable to the estimated number of vortices that passed by Pathfinder per sol during the same season. Phoenix also detected more frequent vortex activity coinciding with passing cold fronts associated to low-pressure baroclinic systems. The clearest example of this was Phoenix sol 95, when 36 pressure drops larger than $0.3 \mathrm{~Pa}$ were identified in contrast to 6 and 7 vortices on the preceding and following sols, respectively. A concurrent cloud feature suggestive of a cold front was seen to cross the Phoenix landing site in images taken by the Mars Color Imager (MARCI) onboard the Mars Reconnaissance Orbiter on that sol (Ellehoj et al. 2010). 
The payload on the Mars Phoenix lander included a mechanical wind sensor, the socalled Telltale (Gunnlaugsson et al. 2008; Holstein-Rathlou et al. 2010), consisting of a lightweight cylindrical mass dangling on a thread attached to a crossbar at the top of the meteorological mast. The Telltale was designed to be deflected by wind and the deflection was observed by imaging the Telltale with the SSI. SSI did not monitor the Telltale continuously. Imaging sequences were implemented during only a limited number of hours per sol, and during these sequences readings were acquired with time intervals longer than 50 seconds (Holstein-Rathlou et al. 2010).

Telltale imaging on nine occasions occurred within 10 seconds of a pressure minimum associated to a passing vortex (Ellehoj et al. 2010). In these events the wind vector was observed to differ by 1.4 to $9.3 \mathrm{~m} \mathrm{~s}^{-1}$ from background wind speeds, magnitudes greater than typical changes between consecutive Telltale images. This observed magnitude range of the wind vector perturbations is in agreement with the range of the observed pressure drops assuming cyclostrophic balance (Ellehoj et al. 2010). Even the strongest vortex-related wind perturbation detected from Telltale measurements, however, is below any estimate of the dust lifting threshold on Mars (Neakrase and Greeley 2010), which is not surprising considering that the derived wind speeds are "snapshots of the wind in random points inside a vortex, not maximum wind speeds, and the great majority of the vortices detected by pressure measurements on Mars are actually too weak to lift dust (Moores et al. 2015; Kahanpää et al. 2016; Steakley and Murphy 2016).

\subsubsection{MSL}

MSL's Rover Environmental Monitoring Station (REMS) includes sensors for pressure, air and ground temperature, wind speed and direction, humidity and UV radiation measurements (Gómez-Elvira et al. 2012). REMS executes five-minute $1 \mathrm{~Hz}$ data acquisition sessions at the start of each LMST hour, with 15 minute and one hour $1 \mathrm{~Hz}$ "extended measurement blocks" implemented at variable times of the sol (Gómez-Elvira et al. 2014). The REMS pressure sensor is, as was MET Phoenix, comprised of Vaisala Barocap ${ }^{\circledR}$ silicon diaphragm sensor heads. REMS' pressure sensor noise is slightly higher (0.2 Pa peak-to-peak) as a result of the faster sampling rate and thus shorter integration time (Harri et al. 2014).

Two major studies have characterized the meteorological signatures of convective vortices identified in the MSL/REMS data (Kahanpää et al. 2016; Steakley and Murphy 2016), identifying dust devils from their temporary pressure declines using slightly different detection criteria. The Kahanpää et al. (2016) criteria are more consistent with the Phoenix dust devil detections (Ellehoj et al. 2010) while the Steakley and Murphy (2016) criteria are more consistent with the Pathfinder dust devil detections (Murphy and Nelli 2002). Despite the different identification criteria both studies come to similar conclusions about vortex activity at Gale Crater.

Few dust devils were anticipated within Gale Crater based upon a lack of observed dust devil tracks and a suggestion of a suppressed atmospheric boundary layer depth (Tyler and Barnes 2013; Haberle et al. 2014). However, approximately 250 pressure vortex signatures were identified during the first Martian year of the mission: Kahanpää et al. (2016) report 252 transient pressure drops with magnitude exceeding $0.5 \mathrm{~Pa}$ during the first 681 sols of the mission, and Steakley and Murphy (2016) report 245 pressure drops with magnitude exceeding $0.3 \mathrm{~Pa}$ during the first 707 sols of the mission. The largest reported pressure drop, $2.97 \mathrm{~Pa}$ and 2.86 a by Kahanpää et al. (2016) and Steakley and Murphy (2016), respectively, was detected on MSL sol 403 at 13:02 Local Mean Solar Time (LMST). Steakley and Murphy's (2016) identification of fewer vortices than Kahanpää et al. (2016) despite their 
lower detection threshold and a $5 \%$ longer study time is partly explained by their criterion that a pressure drop must have a magnitude clearly above background noise. This criterion probably deselects many pressure drops with magnitudes below $0.5 \mathrm{~Pa}$. Also, some larger pressure drops reported by Kahanpää et al. are apparently deselected when local turbulence raises the noise level of the pressure signal. Moreover, Kahanpää et al. (2016) are less strict with the criterion that a pressure drop must have a "dust devil like" shape and they report many pressure events with several minima or otherwise irregular shape.

The Full Width at Half Maximum (FWHM) durations of the pressure drops reported by Kahanpää et al. (2016) follow a distribution almost identical to that reported by Ellehoj et al. (2010) for the Phoenix data, ranging from less than $1 \mathrm{~s}$ to circa $54 \mathrm{~s}$, the mean being circa $9 \mathrm{~s}$. Steakley and Murphy's (2016) FWHM durations range from 1-20 seconds with a median value of 5.3 seconds.

The time-of-sol distribution of MSL's daytime transient pressure drops resembles that detected by Pathfinder and Phoenix, ranging from around 9:00 to 16:00 Local True Solar Time (LTST) with maximum occurrence around noon (Kahanpää et al. 2016; Steakley and Murphy 2016) (Fig. 18). Several night-time pressure 'wiggles' were also identified (Kahanpää et al. 2016). These night-time events exhibit wave-like fluctuations rather than isolated pressure drops and are interpreted as the result of gravity waves initiated by topographic winds (Haberle et al. 2014).

The extended duration of REMS operation provides the first opportunity to assess seasonal vortex occurrence from measured martian pressures. Kahanpää et al. (2016) estimated the mean number of vortices per sol causing pressure drops larger than $0.5 \mathrm{~Pa}$ separately for 8 "seasons" during MSL's first martian year. These estimates ranged from 0.5 per sol $\left(L_{s}\right.$ s $\left.67.5-112.5\right)$ to 1.8 per sol $\left(L_{s}\right.$ 202.5-247.5). Steakley and Murphy (2016) find a spring ( $\left.L_{s} 180-270\right)$ occurrence maximum of 1.5 per sol that is twice the minimum occurrence rate $\left(L_{s} 90-180\right)$. This continuous vortex occurrence through the year is in contrast to the visual MER Spirit imaging observations of detected dust devils only during the "dust devil season" (Greeley et al. 2010, referred in Sect. 2.2.1), but is in rough accordance with the vortex observations performed using the Viking wind data (Ryan and Lucich 1983).

During MSL's first 681 sols there was only one case when more than 3 pressure drops larger than 0.5 Pa were detected within the same LMST hour (Kahanpää et al. 2016). This exception occurred on sol 664 when 16 pressure drops were identified by Kahanpää et al. (2016) between 11:00 and 12:00 LMST. Steakley and Murphy (2016) also identified sol 664 as experiencing the greatest number, four, of verified vortices. This "sol 664 vortex burst" resembles the peaks in vortex activity detected by Phoenix coincident with a passing dust storm front seen in MARCI images.

REMS includes a hot-film anemometer (Domínguez et al. 2008; Gómez-Elvira et al. 2012) designed to distinguish the 3-dimensional wind field. The sensor is mounted on two horizontally aligned booms attached to MSL's Remote Sensing Mast (RSM) which are separated by 120 degrees in azimuth. On each boom there are three identical hot-film anemometer boards sensing wind speed in different directions. Unfortunately, three of the altogether six boards were damaged during MSL's landing (Gómez-Elvira et al. 2014), initiating an ongoing re-calibration effort. To date only 2-dimensional wind measurements have been retrieved (Sara Navarro, Centro de Astrobiología/CSIC-INTA, personal communication, 2015). The compromised wind sensor raw data do qualitatively reveal wind variations concurrent with the transient vortex pressure drops (Kahanpää et al. 2016), with $87 \%$ of the pressure drops being accompanied by wind sensor events. Magnitudes of these wind perturbations could not be determined. 
Available quantified REMS wind measurements consist of derived 5-minute median values. Kahanpää et al. (2016) used these median winds to derive vortex diameters from measured pressure drop durations, assuming that the vortices moved with the velocity of the background (median) wind. Resulting vortex diameters, encompassing pressure perturbation exceeding $0.5 \mathrm{~Pa}$, range from $2.3 \mathrm{~m}$ to $755 \mathrm{~m}$, with a mean of 21 meters and median of 16 meters. The distribution of these is similar to the distribution of dust devil diameters observed by MER Spirit (Greeley et al. 2010).

Solar irradiance loggers have been used terrestrially to detect obscuration of the Sun resulting from dust within a dust devil (Lorenz and Jackson 2015). Compared to camera observations, solar irradiance measurements are better suited for long measurement campaigns with fast sampling rate because of the reduced data volume and wider field of view. REMS' ultraviolet (UV) sensor has been used for a similar survey on Mars (Zorzano et al. 2013). REMS' six UV diodes measure downwelling solar flux in different spectral bands within an upward facing 30-degree half-angle cone field-of-view (Gómez-Elvira et al. 2012). Observations of UV dips coincident with pressure dips are rare. Kahanpää et al. (2016) reported one very weak UV obscuration among its 252 pressure events. Steakley and Murphy (2016) identified 2 pressure events that appear to correspond to UV flux drops, but these pressure events cannot be confirmed as dust devils due to an instrumental error described in Harri et al. (2014) as the shadow effect. Although these events were eliminated from the sample, they may show signs of a double trough signature (Steakley and Murphy 2016) which could be produced by repeated passage into and out of the core of a cylindrical dust devil (Mason et al. 2013). Zorzano et al. (2013) searched for UV obscurations not concurrent with pressure dips, but found no clear signs of dust devils during MSL's first 100 sols. This lack of UV obscurations suggests that most or all vortices detected in the REMS pressure data are dustless, a result that agrees with the detection of only one dust devil by the MSL cameras (Moores et al. 2015).

\section{Discussion}

Despite the differing range of dust devils parameters measured by individual terrestrial campaigns, and their more extensive characterization than has been provided for martian dust devils, comparison between terrestrial and martian dust devils does indicate some differences and some similarities. Martian dust devils exhibit larger maximum widths and heights (Fig. 19) and faster maximum rotational wind speeds than their terrestrial counterparts (see Tables 1 and 2 and references therein). The more extreme inferred martian dust devil winds correspond to what is perceived to be the largest dust devils, which also correspond to the measurement location being most distant from the presumed core location.

Measured terrestrial maximum pressure drops are one order of magnitude larger than the measured maximum martian pressure drops. Renno et al. $(1998,2000)$ suggests that the potential pressure drop magnitude of a dust devil is proportional to the surface pressure. Since surface pressure on Earth is two orders of magnitude greater than on Mars, other aspects affecting potential vortex intensity, including the pressure thickness of the convective layer ('boundary layer height') and the near surface fraction of the mechanical dissipation of energy and the thermodynamic efficiency, must play a role in martian vortices only being one order of magnitude smaller in their depression than terrestrial dust devils. The larger observed size of martian dust devils (Fig. 1) could be one manifestation of these environmental characteristics. 


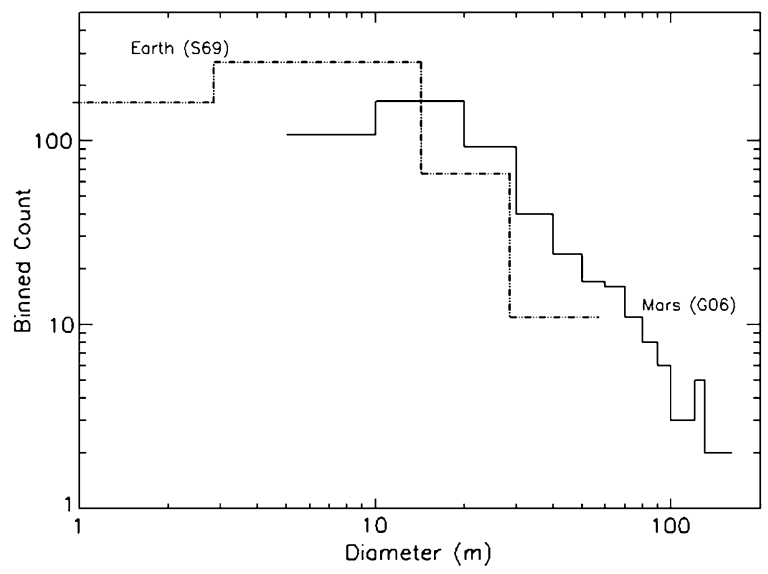

Fig. 19 Differential diameter counts of dust devils from Sinclair's (1969) study at Tucson, and the martian Spirit observations of Greeley et al. (2006) involve roughly comparable survey areas. The roughly linear fall-off towards larger diameters on these logarithmic axes corresponds to a power-law diameter distribution; the turn-over at small diameters may indicate a minimum intrinsic size, or a poor detection efficiency for small devils. In this instance the modal diameter on Earth is more like $5 \mathrm{~m}$, only a factor 3 smaller than on Mars. It is notable that the largest devil seen in the Mars set is larger than that at Earth, but perhaps a longer survey on Earth (pushing the curve upwards) would allow the expected number of detections of larger devils on Earth to rise above unity. As discussed in Lorenz and Jackson (2016) in this issue care must be exercised in drawing size population conclusions from different types of data due to observational biases, especially at the small size end of the distribution

The relative frequency of occurrence of both terrestrial and martian devils with a pressure drop magnitude, $\Delta P$, normalized by the local mean surface pressure exhibits a power law functional form (Fig. 20) represented by, $\Delta P^{-x}$, with $x \sim 2$ (Lorenz 2012a, 2012b). A similar functional form for observed vortex diameter has also been proposed (Lorenz 2009), but an exponential fit has also been suggested, especially at smaller diameters (Fig. 19). These issues are more thoroughly explored in the accompanying papers by Lorenz and Jackson (2016) and Kurgansky et al. (2016).

Terrestrial field measurements have been obtained from numerous geographic locations and continents (Africa, Australia, North America, South America). There are some sites (Eldorado Valley, Nevada USA; Eloy, Arizona USA) that have served as the locales for a number of field campaigns. Martian measurements have also been obtained from discrete locations with a preference (5 of 7 successful missions) for equatorial/subtropical locations, though northern middle and polar latitudes have also been investigated. While martian robotic missions do not offer the opportunity to upgrade or augment instrumentation after launch (and often a substantial time period prior to launch), repeated terrestrial field campaigns to the same location provide the possibility of building upon previous measurements. A number of campaigns over 10-15 years at the Eldorado Valley site have used previously employed and accompanying new instrumentation (Metzger 1999; Balme et al. 2003; Ringrose et al. 2007; Metzger et al. 2010, 2011; Balme et al. 2012; Mason et al. 2014; Jackson and Lorenz 2015). These campaigns have employed mobile and deployable stationary measurement platforms, vertical masts with 10 meter heights to probe the vertical structure of encountered dust devils, stereo camera imaging to improve quantification of dust devil position and size, and recently networks of pressure loggers with partial coverage by solar flux loggers. None of the martian robotic missions to date have 'returned' to a previous lander/rove location, and there is no pronounced motivation to do so for dust 


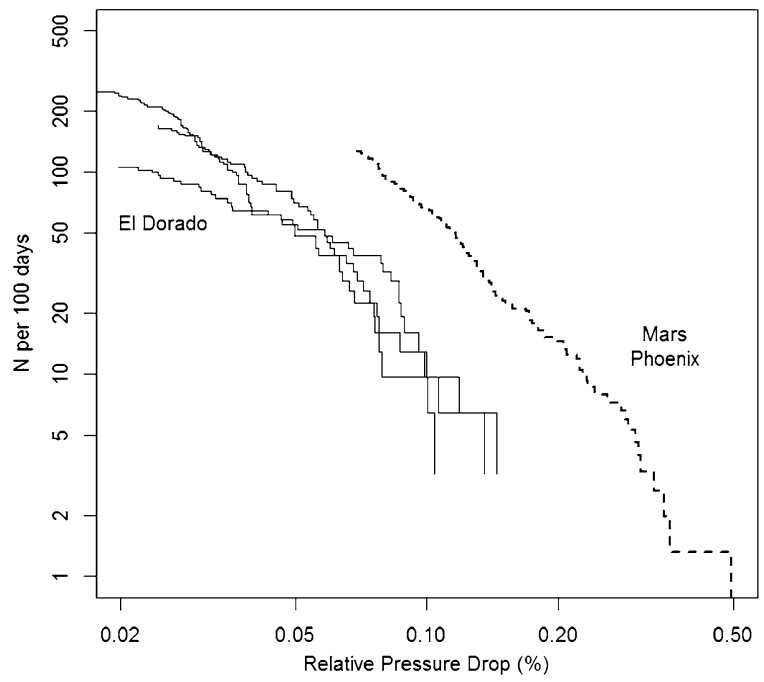

Fig. 20 The number of times a vortex-induced pressure drop will be encountered by a fixed station on Earth and Mars is presented as a cumulative count. Because the absolute pressures on Mars are $>100$ times smaller than on Earth, it is more illuminating to use a relative pressure drop. Thus the 'once a week' vortex on Earth corresponds to about $0.1 \%$ or 1 mbar pressure drop, while that on Mars is about $0.3 \%$ or $\sim 0.02 \mathrm{mbar}(2 \mathrm{~Pa})$. Note that despite the differences in temperature and gas compositions between the two planets, the densities and pressures are approximately in the same proportion. Since the pressure drop of a vortex in cyclostrophic balance is $\sim r V^{2}$, then a fixed relative pressure drop corresponds roughly to a fixed maximum tangential speed $V$ on both bodies: $0.1 \%$ for a $10 \mathrm{~m} / \mathrm{s}$ wind speed

devil measurements. One opportunity to do so could be realized with a Mars sample return set of missions which could have a second spacecraft retrieve a sample previously cached by a preliminary mission. If the preliminary mission's measurements identified unique dust devil characteristics at the cache site, possibly the cache retrieving mission could carry instrumentation to enhance characterization of the dust devils at the cache site, but mission lead time development needs and other mission priorities would likely preclude such a an instrumentation selection.

\section{Future Dust Devil Measurements}

\subsection{Earth}

The basic features and correlations of properties of terrestrial dust devils have been measured, and somewhat robust statistics on the population are emerging. What is presently lacking are data to support an evaluation of the dependence of dust devil morphology on environmental parameters. For example, there is a somewhat anecdotal (see Chap. 6, Kurgansky et al. 2016) correlation of columnar vortices with very smooth land surfaces such as playa, whereas rougher (e.g. scrubby) terrain tends to have more conical devils.

Present profiles of dust devils do not allow discrimination of the various analytic functions used as axisymmetric idealizations of vortex structure (Kurgansky et al. 2016). On the other hand, it is not clear that better measurements will help, as it may be that nonidealities are more significant than the difference between models. A prominent area that merits 
further study is the frequency and nature of multi-cored vortices and what controls their formation. Another deviation from axisymmetry is evident in simulations (e.g. Toigo et al. 2003) - that winds spiral into the vortex core in azimuthally-concentrated bands, like those seen in hurricane clouds or in the arms of galaxies. Such azimuth variations have not yet been formally documented in field observations. Finally, field measurements from Sinclair (1973) and Lorenz (2012b) appear to show an asymmetry in the pressure profile of migrating dust devils, the 'attack' slope being shallower than the 'decay'. This may be a generic feature of a migrating vortex, tilted by wind shear, but has not been quantified.

Another question is the possible role in dust devil energy balance of sunlight intercepted by lofted dust. It is sometimes noticed that devils become more intense when they become dustier-but is this cause, or effect? If effect, is it sensible heat in the dust and air pulled off the ground, or is the solar heat introduced by the absorbing dust and introduced into the vortex directly a significant factor? Field measurements, perhaps including thermal as well as video imaging (to relate flow speeds to dust temperature) may illuminate this question.

Related to this question is that of dust-lifting per-se: do larger, more vigorous, but less frequently occurring, dust devils lift more dust than smaller, more numerous ones? Can the dust lifting of a dust devil ever be disentangled from the properties of the surface (i.e. is the dustiness of a dust devil controlled mainly be surface properties or by its internal properties?). Only by coordinated measurements of dust content and wind speed (likely in-situ measurement), and dust devil diameter (likely remote measurements) from controlled field-sites can these questions be addressed. Such measurements would help provide better understanding of the environmental effects of dust devils and provide a model for comparison with Mars.

Circumstantial observations, dating as far back as Flower (1936) and a correlation of satellite-observed dust devil heights and estimates of planetary boundary layer thickness from model predictions and occultation measurements (Fenton and Lorenz 2015) suggest dust devil heights and spacings may be controlled by the planetary boundary layer (PBL) thickness. In-situ measurements that establish this correlation more securely (perhaps also validating the anecdotal suggestion that small devils are more common early in the day and larger ones in the late afternoon, consistent with the diurnal growth of the PBL) would similarly be useful.

In summary, terrestrial dust devil characterization would benefit from dedicated campaigns of long-lived networks of uniformly instrumented stations measuring pressure, wind (3-D), temperature, dust load, electric field, etc. Measurements at a variety of heights above the surface would be valuable. High cadence $(1 \mathrm{~Hz})$ measurements in conjunction with imaging of the network would aid in correlating visible dust devil manifestation with interior conditions. The use of several networks in dissimilar field sites would enable the influence of local surface properties to be disentangled from the properties of individual dust devils.

\subsection{Mars}

Measurement characterization of martian dust devils is limited by the scarcity of observations, especially those that provide simultaneous measurements of a variety of geophysical parameters. Since dust devils play a substantial role in maintaining Mars' persistent dust haze, especially during the aphelion season, better characterizing their attributes will enable improved understanding of the their role in providing/maintaining the thermodynamically important atmospheric dust load.

Robotic spacecraft sent to Mars' surface should carry imaging systems capable of providing high resolution and fast frame rates covering a wide field of view. Monochrome imaging is adequate (Greeley et al. 2010) and would not interfere with other onboard color 
imagers dedicated to geologic studies. An imaging system that provides full azimuthal coverage and onboard software to identify and retain images that display dust devil diagnostic temporal variability (thus reducing downlink volume of dust-devil deficient images) would enable greater temporal coverage than has been provided previously. The dust-devil imaging strategy employed by Spirit (and Opportunity) Navigation Cameras did provide substantial numbers of dust devil observations, targeted to the times of sol when they were most frequent.

No robotic spacecraft has yet provided unambiguous contemporaneous measurement of surface pressure and wind. Thus, the dynamic connection between vortex magnitude and velocity fields has not been established. Hot wire / hot film anemometers have been the only type of high-frequency sampling wind instruments to operate on Mars' surface, and all have suffered from instrument failure and/or calibration difficulties and placement issues (Lenoir et al. 2011; Lorenz and Sotzen 2014). These instruments have generally been low mass, which are desirable, but their poor performance record suggests other sensor types might be warranted. An enhanced REMS wind sensor is selected for NASA's InSight lander (scheduled for a 2018 launch). NASA's 2020 Mars Rover will carry a meteorology package (Rodriguez-Manfredi et al. 2014). Acoustic and laser Doppler anemometers have been suggested as possible technologies applicable for Mars (Merrison et al. 2006; Wilson et al. 2008; Esposito 2011; Banfield 2012; Leonard-Pugh et al. 2012; Rafkin et al. 2013). Both technologies enable high frequency sampling, $10 \mathrm{~Hz}$ or more, of the threedimensional wind field, which provides opportunity for determination of turbulent characteristics of the flow. Acoustic anemometry has the added advantage of providing simultaneous atmospheric temperature measurement which when coupled to the winds affords quantification of the turbulent thermal flux. To date neither technology has been selected for flight. Wind socks provide wind sampling at very low frequencies (Sullivan et al. 2000; Holstein-Rathlou et al. 2010), but their data bandwidth requirement is large compared to their low response time and inability to unambiguously provide the vertical wind component.

Pressure measurements have provided the most valuable and plentiful in situ dust devil/convective vortex measurements to date. The high resolution $(0.1-0.2 \mathrm{~Pa})$ and high sampling frequencies $(2-0.25 \mathrm{~Hz})$ implemented have proved adequate to capture pressure signatures. Care must be taken for future opportunities to avoid sensor thermal variation which complicates pressure value determination, as well as inlet orientation which can provide unfavorable dynamic pressure contamination, and measurement tube length/diameter which affects sensor response time (Ellehoj et al. 2010). The low power and mass requirements of the necessary equipment as well as the tremendous value of martian surface pressure measurements for both local (dust devil) and larger scale atmospheric analyses warrant pressure sensors being included on every robotic spacecraft landed upon Mars' surface.

Contemporaneous measurement of received solar flux and pressure/wind signatures of vortex passage are necessary to assess the 'dustiness' of vortices and thus the frequency of occurrence of dust devils vs. 'clear' convective vortices. Such discrimination would aid the assessment of vortex thermal vs. dust vertical fluxes. Dedicated solar flux instruments can provide appropriate measurements (Gómez-Elvira et al. 2012; Kahanpää et al. 2016) with a cadence coupled to that of other science instruments. Engineering systems can also provide solar flux measurements at no additional 'cost (Schofield et al. 1997), though likely at a lower cadence than desired for science purposes.

To date no martian robotic lander/rover has provided electric field or dust concentration or dust particle flux/size distribution measurements. ESA's Schiaparelli lander's MicroARES system will provide initial electric field measurements during that missions short 
duration operations in late 2016 (Esposito et al. 2016). A laser Doppler anemometer could provide particle concentration in addition to velocity, but as previously stated no such instrument is currently selected for a Mars robotic mission. A vertically oriented LIDAR system onboard the NASA Phoenix lander provided vertical resolution of aerosol concentration within the bottom few kilometers of the atmosphere (Whiteway et al. 2008). A horizontally scanning LIDAR system could provide signals from which radial variations in near-surface aerosol abundance within several kilometers of a lander/rover could be obtained.

Dust devil infrasound/acoustic and seismic measurements are still minimally available for terrestrial studies, and their full diagnostic value remains immature yet promising; a seismometer, for example, appears to be capable of tracking close encounters with dust devils, recovering an estimate of the azimuth history and constraining the integral of the pressure field (relating to diameter and core pressure drop). In combination with wind and pressure measurements, the dust devil parameters and miss distance may be reconstructed or at least constrained (Lorenz et al. 2015a). The NASA InSight mission will provide meteorological and seismic measurements that will improve understanding of dust devils for both martian and terrestrial applications. In addition, the seismic signals of dust devils may also provide information about the surface and subsurface properties at the InSight landing site by acting as calibration loads (Lorenz et al. 2015a, 2015b). A Mars microphone is going to be part of the payload of the 2018 Exomars landing platform (Zelenyi et al. 2015). The microphone, with a bandwidth of $100 \mathrm{~Hz}$ to $10 \mathrm{kHz}$, will record, for the first time, audio signals at the surface of Mars and is expected to contribute to atmospheric science investigations including dust devil studies (Maurice et al. 2016).

Ideally a network of meteorologically outfitted stations designed to characterize dust devils will be deployed upon Mars' surface within a very limited spatial footprint, possibly as one component of a more globally extensive network. The PASCAL network mission developed and proposed to from NASA Ames in the late 1990's/early 2000's developed some network concepts but was not selected to launch.

\section{Conclusions}

Dust devils are inherently difficult to measure. Their occurrence is somewhat unpredictable in both space and time and they are fast-moving, short-lived phenomena. Observational 'survey' data have provided information about size-frequency distributions, sense of rotation and other parameters, but to assess the wind and pressure structure, and the dust-loading behaviour of a dust devil has required in-situ measurement.

To obtain these data, terrestrial dust devils have either been "chased" or "monitored". The former method can be subject to observation bias, but the latter can require long field campaigns and multiple sets of instruments if statistically significant numbers of data are to be obtained. Recently, advances in both instrumentation and data recording technology have enabled new methodologies to be developed to solve these problems. Long-term, high cadence monitoring using multiple sensors, coupled with automatically controlled imaging provides a way to get the 'best of both worlds'. In the next ten years, new field studies should be able to provide much better data about population-statistics of dust devils and their environmental role.

On Mars, dust devils studies have generally 'piggy-backed' on other camera or meteorological studies. Nevertheless, significant progress in measuring population statistics and pressure-structure have been possible. These data have been complemented by remotesensing observations from orbit—a method not widely applied on Earth. 
Future studies for Mars should focus on in situ, high cadence sampling of pressure, wind speed and dust load. Along with the continuing operation of the US/NASA MSL Curiosity rover, the next opportunity for martian dust devil measurements will be provided by the ESA/Russian ExoMars Schiaparelli Lander scheduled for $\sim 1$ week's operation on Mars surface during late 2016. The longer lived US/NASA InSight lander is scheduled to arrive in late 2018, followed by the ESA ExoMars Rover and the US/NASA 2020 Rover in early 2021.

Acknowledgements All authors thank the International Space Science Institute for organizing and hosting the February 2015 Bern, Switzerland Workshop and for providing lodging accommodations. J. Murphy thanks the New Mexico State University College of Arts \& Sciences Faculty Travel Award program for travel support. K. Steakley thanks the New Mexico State University (NMSU) Aggies Go Global organization, NMSU Graduate School, Associated Students of NMSU, New Mexico Space Grant Consortium, and NMSU Astronomy Department for funding support. M. Balme acknowledges funding from the UK Space Agency (grant ST/L00643X/1) and from STFC, the UK Science and Technology Facilities Council (grant ST/L000776/1). R. Lorenz acknowledges the support of NASA Mars Fundamental Research Program Grant NNX12AI04G. N. Murdoch was supported by a CNES-provided Post-doctoral award. M. Patel acknowledges support from the UK STFC and UK Space Agency under grants ST/I003061/1 and ST/P001262/1 and as part of the project UPWARDS-633127, funded by the European Union's Horizon 2020 Programme (H2020-Compet-08-2014). P. Whelley was supported by the NASA Post Doctoral Program and NASA's Remote, In Situ, and Synchrotron Studies for Science and Exploration project.

The authors thank two anonymous reviewers for their thorough evaluations which have resulted in an improved final paper.

\section{References}

R.A. Bagnold, The Physics of Blown Sand and Desert Dunes (Dover, Mineola, 1941)

M.R. Balme, A. Pathare, S.M. Metzger, M.C. Towner, S.R. Lewis, A. Spiga, L.K. Fenton, N.O. Renno, H.M. Elliott, F.A. Saca, T.I. Michaels, P. Russell, J. Verdasca, Field measurements of horizontal forward motion velocities of terrestrial dust devils: towards a proxy for ambient winds on Mars and Earth. Icarus 221(2), 632-645 (2012)

M. Balme, R. Greeley, Dust devils on Earth and Mars. Rev. Geophys. 44, RG3003 (2006)

M. Balme, S. Metzger, M. Towner, T. Ringrose, R. Greeley, J. Iversen, Friction wind speeds in dust devils: a field study. Geophys. Res. Lett. 30(16), 1830 (2003). doi:10.1029/2003GL017493

D. Banfield, Mars acoustic anemometer, AGU Fall Meeting 2012, San Francisco, California, USA. Poster presentation (2012), http://abstractsearch.agu.org/meetings/2012/FM/P23A-1916.html

D. Banfield, Atmospheric observations from the Mars insight mission, in Fifth International Workshop on the Mars Atmosphere: Modelling and Observations, Oxford, UK (2014). Oral presentation, http:// www-mars.lmd.jussieu.fr/oxford2014/abstracts/banfield_oxford2014.pdf

A.J. Bedard, Low frequency atmospheric acoustic energy associated with vortices produced by thunderstorms. Mon. Weather Rev. 241-243 (2005)

F. Bell, Dust devils and aviation, report, Meteorol. Note 27, Aust. Bur. of Meteorol., Melbourne, Victoria, 1967

J.-J. Berthelier, ARES, atmospheric relaxation and electric field sensor, the electric field experiment on NETLANDER. Planet. Space Sci. 48(12-14), 1193-2000 (2000)

C. Bettanini et al., The DREAMS experiment on the ExoMars 2016 mission for the study of martian environment during the dust storm season. MetroAeroSpace 167(173), 29-30 (2014). doi:10.1109/ MetroAeroSpace.2014.6865914

H.B. Bluestein, A.L. Pazmany, Observations of tornadoes and other convective phenomena with a mobile, 3-mm wavelength, Doppler radar: the spring 1999 field experiment. Bull. Am. Meteorol. Soc. 81, 2939$2951(2000)$

J.J. Carroll, J.A. Ryan, Atmospheric vorticity and dust devil rotation. J. Geophys. Res. 75, 5179-5184 (1970)

W.D. Crozier, Dust devil properties. J. Geophys. Res. 75, 4583-4585 (1970)

R. Davy, J.A. Davies, P.A. Taylor, C. Lange, W. Weng, J. Whiteway, H.P. Gunnlaugson, Initial analysis of air temperature and related data from the Phoenix MET station and their use in estimating turbulent heat fluxes. J. Geophys. Res. 115, E00E13 (2010). doi:10.1029/2009JE003444 
S.J. Desch, J.N. Cuzzi, The generation of lightning in the solar nebula. Icarus 143, 87-105 (2000). doi: 10.1006/icar.1999.6245

M. Domínguez, V. Jiménez, J. Ricart, L. Kowalski, J. Torres, S. Navarro, J. Romeral, L. Castañer, A hot film anemometer for the martian atmosphere. Planet. Space Sci. 56(8), 1169-1179 (2008). doi:10.1016/ j.pss.2008.02.013

N. Duff, D.J. Lacks, Particle dynamics simulations of triboelectric charging in granular insulator systems. J. Electrost. 66, 51 (2008). doi:10.1016/j.elstat.2007.08.005

R. Edmonds, Examination of two martian atmosphere phenomena: dust devil acoustics and gravity wave forcing of dust storm development. PhD dissertation, New Mexico State University, 2014, p. 223

M.D. Ellehoj, H.P. Gunnlaugsson, P.A. Taylor, H. Kahanpää, K.M. Bean, B.A. Cantor, B.T. Gheynani, L. Drube, D. Fisher, A.-M. Harri, C. Holstein-Rathlou, M.T. Lemmon, M.B. Madsen, M.C. Malin, J. Polkko, P.H. Smith, L.K. Tamppari, W. Weng, J. Whiteway, Convective vortices and dust devils as the Phoenix Mars mission landing site. J. Geophys. Res. 115, E00E16 (2010). doi:10.1029/2009JE003413

F. Esposito, MEDUSA: observation of atmospheric dust and water vapor close to the surface of Mars. Mars 6, 1-12 (2011). http://www.marsjournal.org/contents/2011/0001/. doi:10.1555/mars.2011.0001

F. Esposito, R. Molinaro, C.I. Popa, C. Molfese, F. Cozzolino, L. Marty, K. Taj-Eddine, G. Di Achille, G. Franzese, S. Silvestro, G.C. Ori, The role of atmospheric electric field in the dust lifting process. Geophys. Res. Lett. (2016, in press)

L.K. Fenton, R. Lorenz, Dust devil height and spacing with relation to the martian planetary boundary layer thickness. Icarus 260, 246-262 (2015). doi:10.1016/j.icarus2015.07.028

L. Fenton, D. Reiss, M. Lemmon, B. Marticorena, S. Lewis, B. Cantor, Orbital observations of dust lofted by daytime convective turbulence. Space Sci. Rev. (2016). doi:10.1007/s11214-016-0243-6

F. Ferri, P.H. Smith, M.T. Lemmon, N. Renno, Dust devils as observed by Mars Pathfinder. J. Geophys. Res. 108(E12), 5133 (2003). doi:10.1029/2000JE001421

D.E. Fitzjarrald, A field investigation of dust devils. J. Appl. Meteorol. 12, 808-813 (1973)

W.D. Flower, Sand devils. Lond. Meteorol. Off. Prof. Notes 5(71), 1-16 (1936)

K.M. Forward, D.J. Lacks, R.M. Sankaran, Particle-size dependent bipolar charging of martian regolith simulant. Geophys. Res. Lett. 36(13), L13201 (2009)

G.D. Freier, The electric field of a large dust devil. J. Geophys. Res. 65, 3504 (1960). doi:10.1029/ JZ065i010p03504

S.D. Fuerstenau, Solar heating of suspended particles and the dynamics of martian dust devils. Geophys. Res. Lett. 33, 19 (2006). doi:10.1029/2006GL026798

E.W.B. Gill, Friction electrification of sand. Nature 162, 568 (1948)

D.A. Gillette, P. Sinclair, Estimation of suspension of alkaline material by dust devils in the United States. Atmos. Environ. 24(A), 1135-1142 (1990)

D.A. Gillette, I.H. Blifford, D.W. Fryrear, Influence of wind velocity on size distributions of aerosols generated by wind erosion of soils. J. Geophys. Res. 79, 4068-4075 (1974)

M. Golombek et al., Overview of the Mars Pathfinder mission and assessment of landing site predictions. Science 278, 5344 (1997). doi:10.1126/science.278.5344.1743

J. Gómez-Elvira et al., REMS: the environmental sensor suite for the Mars science laboratory rover. Space Sci. Rev. 170, 583-640 (2012). doi:10.1007/s11214-012-9921-1

J. Gómez-Elvira et al., Curiosity's rover environmental monitoring station: overview of the first 100 sols. J. Geophys. Res., Planets 119, 1680-1688 (2014). doi:10.1002/2013JE004576

C.G. Grant, Dust devils in the sub-arctic. Weather 4, 402-403 (1949)

R. Greeley, J.D. Iversen, Wind as a Geological Process. Cambridge Planetary Science Series (Cambridge University Press, Cambridge, 1985). ISBN 0521356927

R. Greeley et al., Wind related features in Gusev crater, Mars. J. Geophys. Res. 108(E12), 8077 (2003)

R. Greeley, P.L. Whelley, R.E. Arvidson, N.A. Cabrol, D.J. Foley, B.J. Franklin, P.G. Geissler, M.P. Golombek, R.O. Kuzmin, G.A. Landis, M.T. Lemmon, L.D.V. Neakrase, S.W. Squyres, S.D. Thompson, Active dust devils in Gusev Crater, Mars: observations from the Mars exploration rover spirit. J. Geophys. Res. 111(E12) (2006). doi:10.1029/2006JE002743

R. Greeley, D.A. Waller, N.A. Cabrol, G.A. Landis, M.T. Lemmon, L.V. Nekarase, M. Pendelton Hoffer, S.D. Thompson, Gusev Crater, Mars: observations of three dust devil seasons. J. Geophys. Res. 115 (2010)

J. Grotzinger et al., Mars science laboratory mission and science investigation. Space Sci. Rev. 170(1), 5-56 (2012). doi:10.1007/s11214-012-9892-2

H.P. Gunnlaugsson et al., Telltale wind indicator for the Mars Phoenix lander. J. Geophys. Res. 113, E00A04 (2008). doi:10.1029/2007JE003008

R.M. Haberle, J. Gómez-Elvira, M. de la Torre Juárez, A.-M. Harri, J.L. Hollingsworth, H. Kahanpää, M.A. Kahre, M. Lemmon, F.J. Martín-Torres, M. Mischna, J.E. Moores, C. Newman, S.C.R. Rafkin, N. Rennó, M.I. Richardson, J.A. Rodríguez-Manfredi, A.R. Vasavada, M.-P. Zorzano-Mier, REMS/MSL Science Teams, Preliminary interpretation of the REMS pressure data from the first 100 sols of the MSL mission. J. Geophys. Res., Planets 119, 440-453 (2014). doi:10.1002/2013JE004488 
J. Hallett, T. Hoffer, Dust devil systems. Weather 26, 247-250 (1971)

W.R. Harper, Contact and Frictional Dissipation (Clarendon Press, Oxford, 1967)

A.-M. Harri, M. Genzer, O. Kemppinen, H. Kahanpää, J. Gomez-Elvira, J.A. Rodriguez-Manfredi, R. Haberle, J. Polkko, W. Schmidt, H. Savijärvi, J. Kauhanen, E. Atlaskin, M. Richardson, T. Siili, M. Paton, M. de la Torre Juarez, C. Newman, S. Rafkin, M.T. Lemmon, M. Mischna, S. Merikallio, H. Haukka, J. Martin-Torres, M.-P. Zorzano, V. Peinado, R. Urqui, A. Lapinette, A. Scodary, T. Mäkinen, L. Vazquez, N. Rennó, The REMS/MSL Science Team, Pressure observations by the curiosity rover: initial results. J. Geophys. Res., Planets 119, 82-92 (2014). doi:10.1002/2013JE004423

R.G. Harrison, E. Barth, F. Esposito, J. Merrison, F. Montmessin, K.L. Aplin, C. Borlina, J.J. Berthelier, G. Déprez, W.M. Farrell, I.M.P. Houghton, N.O. Renno, K.A. Nicoll, S.N. Tripathi, M. Zimmerman, Applications of electrified dust and dust devil electrodynamics to martian atmospheric electricity. Space Sci. Rev. (2016). doi:10.1007/s11214-016-0241-8

M. Hecht, M.D. Tratt, D. Catling, S. Samulon, MATADOR dust devil campaign (2001)

G.D. Hess, K.T. Spillane, Characteristics of dust devils in Australia. J. Appl. Meteorol. 29, 498-507 (1990)

S.L. Hess, R.M. Henry, C.B. Leovy, J.A. Ryan, J.E. Tillman, Meteorological results from the surface of Mars: viking 1 and 2. J. Geophys. Res. 82(28), 4559-4574 (1977). doi:10.1029/JS082i028p04559

C. Holstein-Rathlou et al., Winds at the Phoenix landing site. J. Geophys. Res. 115, E00E18 (2010). doi: 10.1029/2009JE003411

R.G. Horn, D.T. Smith, A. Grabbe, Contact electrification induced by monolayer modification of a surface and relation to acid-base interactions. Nature 366, 442-443 (1993). doi:10.1038/366442a0

M.S. Howe, Theory of Vortex Sound (Cambridge University Press, Cambridge, 2003)

I.I. Inculet, G.S.P. Castle, G. Aartsen, Generation of bipolar electric fields during industrial handling of powders. Chem. Eng. Sci. 61, 2249-2253 (2006). doi:10.1016/j.ces.2005.05.005

J. Ito, H. Niino, Particle image velocimetry of a dust devil observed in a desert. Sci. Online Lett. Atmos. 10(1), 108-111 (2014)

R.L. Ives, Behavior of dust devils. Bull. Am. Meteorol. Soc. 28, 168-174 (1947)

D.J. Lacks, A. Levandovsky, Effect of particle size distribution on the polarity of triboelectric charging in granular insulator systems. J. Electrost. 65, 107 (2007)

T.L. Jackson, W.M. Farrell, IEEE Trans. Geosci. Remote Sens. 44, 2942 (2006). doi:10.1109/TGRS.2006. 875785

B. Jackson, R. Lorenz, A multiyear dust devil vortex survey using an automated search of pressure time series. J. Geophys. Res. 120(2), 401-412 (2015). doi:10.1092/2014JE004712

H. Kahanpää, C. Newman, J. Moores, M-P. Zorzano, J. Martín-Torres, S. Navarro, A. Lepinette, M.T. Lemmon, B. Cantor, P. Valentín-Serrano, A. Ullán, W. Schmidt, Convective vortices and dust devils at the MSL landing site: annual variability. J. Geophys. Res. Planets 121 (2016). doi:10.1002/2016JE005027

M. Kahre, J. Murphy, R. Haberle, Modeling the martian dust cycle and surface dust reservoirs with the NASA Ames general circulation model. J. Geophys. Res., Planets 111, E6 (2006)

J.C. Kaimal, J.A. Bussinger, Case studies of a convective plume and a dust devil. J. Appl. Meteorol. 9, 612$620(1970)$

M. Klose, B.C. Jemmet-Smith, H. Kahanpää, M. Kahre, P. Knippertz, M. Lemmon, S. Lewis, R. Lorenz, L. Neakrase, C. Newman, M. Patel, D. Reiss, A. Spioga, P. Whelley, Space Sci. Rev. (2016). doi:10.1007/ s11214-016-0261-4

J.F. Kok, N.O. Renno, Enhancement of the emission of mineral dust aerosols by electric forces. Geophys. Res. Lett. 33, L19S10 (2006)

J.F. Kok, N.O. Renno, Electrostatics in wind-blown sand. Phys. Rev. Lett. 100, 014501 (2008)

M.V. Kurgansky, Size distribution of dust devils in the atmosphere. Izv., Atmos. Ocean. Phys. 42(3), 319-325 (2006)

M.V. Kurgansky et al., Dust devil steady-state structure from a fluid dynamic perspective. Space Sci. Rev. (2016). doi:10.1007/s11214-016-0281-0

M.V. Kurgansky, A. Montecinos, V. Villagran, S.M. Metzger, Micrometeorological conditions for dustdevil occurrence in the Atacama Desert. Bound.-Layer Meteorol. 138, 285-298 (2011). doi:10.1007/ s10546-010-9549-1

R.I. Lambeth, On the measurement of dust devil parameters. Bull. Am. Meteorol. Soc. 47, 522-526 (1966)

G.A. Landis, P.P. Jenkins, Measurement of the settling rate of atmospheric dust on Mars by the MAE instrument on Mars Pathfinder. J. Geophys. Res. 105(E1), 1855-1857 (2000). doi:10.1029/1999JE001029

J. Latham, The electrification of snowstorms and sandstorms. Q. J. R. Meteorol. Soc. 90, 383 (1964). doi: 10.1002/qj.49709038310

J. Latham, C.D. Stow, A laboratory investigation of the electrification of snowstorms. Q. J. R. Meteorol. Soc. 94, $415(1968)$ 
M.T. Lemmon, M.J. Wolff, M.D. Smith, R.T. Clancy, D. Banfield, G.A. Landis, A. Ghosh, P.H. Smith, N. Spanovich, B. Whitney, P. Whelley, R. Greeley, S. Thompson, J.F. Bell III, S.W. Squyres, Atmospheric imaging results from the Mars exploration rovers: spirit and opportunity. Science 306, 17531756 (2004)

M.T. Lemmon, M.J. Wolff, J.F. Bell III., M.D. Smith, B.A. Cantor, P.H. Smith, Dust aerosol, clouds and the atmospheric optical depth record over 5 Mars years of the Mars exploration rover mission. Icarus 251, 96-111 (2015)

B. Lenoir, D. Banfield, D.A. Caughey, Accommodation study for an anemometer on a martian lander. J. Atmos. Ocean. Technol. 28, 210-218 (2011). doi:10.1175/2010JTECHA1490.1

E. Leonard-Pugh, C. Wilson, S. Calcutt, L. Davis, Capacitive ultrasonic transducer development for acoustic anemometry on Mars in 44th Annual Meeting of the Division for Planetary Sciences of the American Astronomical Society, Reno, NV, USA (2012). Poster presentation, http://adsabs.harvard.edu/ abs/2012DPS....4421523L

P. Lognonné, B. Mosser, Planetary seismology. Surv. Geophys. 14, 239-302 (1993)

P. Lognonné, W.B. Banerdt, K. Hurst, D. Mimoun, R. Garcia, M. Lefeuvre, J. Gagnepain-Beyneix, M. Wieczorek, A. Mocquet, M. Panning, E. Beucler, S. Deraucourt, D. Giardini, L. Boschi, U. Christensen, W. Goetz, T. Pike, C. Johnson, R. Weber, K. Larmat, N. Kobayashi, J. Tromp, Insight and single-station broadband seismology: from signal and noise to interior structure determination, in 43rd Lunar and Planetary Science Conference, Houston, TX, March 2012 (2012). Abstract \#1983

R. Lorenz, Thermal imaging of a desert dust devil. J. Meteorol. 29(292), 275-276 (2004)

R.D. Lorenz, Power law of dust devil diameters on Mars and Earth. Icarus 203(2), 683-684 (2009)

R. Lorenz, On the statistical distribution of dust devil diameter. Icarus 215(1), 381-390 (2011)

R.D. Lorenz, Power law distribution of pressure drops in dust devils: observation techniques and Earth-Mars comparison. Planet. Space Sci. 60, 370-375 (2012a). doi:10.1016/j.pss.2011.11.003

R.D. Lorenz, Observing desert dust devils with a pressure logger. Geosci. Instrum. Method. Data Syst. 1, 209-220 (2012b)

R. Lorenz, The longevity and aspect ratio of dust devils: effects on detection efficiencies and comparison of landed and orbital imaging at Mars. Icarus 226, 964-970 (2013)

R.D. Lorenz, Vortex encounter rates with fixed barometer stations: comparison with visual dust devil counts and large eddy simulations. J. Atmos. Sci. 71, 4461-4472 (2014)

R.D. Lorenz, Heuristic estimation of dust devil vortex parameters and trajectories from single-station meteorological observations: application to InSight at Mars. Icarus 271(2-16), 326-337 (2016)

R.D. Lorenz, D. Christie, Dust devil signatures in infrasound records of the international monitoring system. Geophys. Res. Lett. 42(6), 2009-2014 (2015)

R.D. Lorenz, B.K. Jackson, Dust devils and dustless vortices on a desert playa observed with surface pressure and solar flux logging. GeoResJ 5, 1-11 (2015). doi:10.1016/j.grj.2014.11.002

R.D. Lorenz, B. Jackson, Dust devil populations and statistics. Space Sci. Rev. (2016). doi:10.1007/s11214016-0277-9

R.D. Lorenz, P.D. Lanagan, A barometric survey of dust devil vortices on a desert playa. Bound.-Layer Meteorol. 53, 555-568 (2014). doi:10.1007/s10546-014-9954-y

R. Lorenz, J. Radebaugh, Dust devils in thin air: vortex observations at a high elevation Mars analog site in the Argentinian Puna. Geophys. Res. Lett. 43 (2016). doi:10.1002/2015GL067412

R.D. Lorenz, D. Reiss, Solar panel clearing events, dust devil tracks, and in-situ vortex detections on Mars. Icarus 248, 162-164 (2015)

R.D. Lorenz, K.S. Sotzen, Buoyant thermal plumes from planetary landers and rovers: application to sizing of meteorological masts. Planet. Space Sci. 90, 81-89 (2014). doi:10.1016/j.pss.2013.10.011

R.D. Lorenz, S. Kedar, N. Murdoch, P. Lognonné, T. Kawamurak, D. Mimoun, W.B. Banerdt, Seismometer signature of dust devils: implication for InSight, in 2015 European and Planetary Science Conference, Nantes (2015a)

R.D. Lorenz, S. Kedar, N. Murdoch, P. Lognonné, T. Kawamurak, D. Mimoun, W.B. Banerdt, Seismometer detection of dust devil vortices by ground tilt. Bull. Seismol. Soc. Am. BSSA-S-15-00169 (2015b)

R.D. Lorenz, L.D. Neakrase, J.D. Anderson, In-situ measurement of dust devil activity at La Jornada Experimental Range, New Mexico, USA. Aeolian Res. 19, 183-194 (2015c)

R.D. Lorenz, M. Balme, Z. Gu et al., History and application of dust devil studies. Space Sci. Rev. (2016). doi:10.1007/s11214-016-0239-2

J. Lowell, W.S. Truscott, Triboelectrification of identical insulators. II. Theory and further experiments. J. Phys. D, Appl. Phys. 19, 1281-1298 (1986). doi:10.1088/0022-3727/19/7/018

J.P. Mason, M.R. Patel, S.R. Lewis, Radiative transfer modelling of dust devils. Icarus 223, 1-10 (2013)

J.P. Mason, M.R. Patel, S.W. Lewis, The retrieval of optical properties from terrestrial dust devil vortices. Icarus 231, 385-393 (2014) 
S. Maurice, R.C. Wiens, W. Rapin, D. Mimoun, X. Jacob, B. Betts, S. Clegg, A. Cousin, O. Gasnault, O. Forni, J. Lasue, P.-Y. Meslin, J.F. Bell, G. Delory, A microphone supporting LIBS investigation on Mars. Lunar Planet. Sci. Conf. 47, 3044 (2016)

J.P. Merrison, H.P. Gunnlaugsson, K. Kinch, T.L. Jacobsen, A.E. Jensen, P. Nørnberg, H. Wahlgreen, An integrated laser anemometer and dust accumulator for studying wind-induced dust transport on Mars. Planet. Space Sci. 54(11), 1065-1072 (2006). doi:10.1016/j.pss.2006.05.026

S.M. Metzger, Dust devils as aeolian transport mechanisms in southern Nevada and in the Mars Pathfinder landing site. Ph.D. thesis, Univ. of Nev., Reno (1999)

S.M. Metzger, J.R. Carr, J.R. Johnson, T.J. Parker, M.T. Lemmon, Dust devil vortices seen by the Mars Pathfinder camera. Geophys. Res. Lett. 26(18), 2781-2784 (1999)

S.M. Metzger, M. Kurgansky, A. Montecinos, V. Villagram, H. Verdejo, Chasing dust devils in Chile's Atacama Desert. LPSC Abstract 2564 (2010)

S. Metzger, M. Balme, A. Pathare, N. Renno, M. Towner, A. Spiga, H. Elliott, High-resolution dust devil sampling for sediment loads, wind speeds, temperature and pressure excursions, in 42nd Lunar and Planetary Science Conference. Texas (2011). Abstr. \#1608

G.J. Molina-Cuberos et al., A new approach for estimating Titan's electrical conductivity based on data from relaxation probe sensors on the Huygens experiment. Planet. Space Sci. 58(14-15) (2010). doi:10.1016/j.pss.2010.09.014

J.E. Moores et al., Observational evidence of a suppressed planetary boundary layer in northern Gale Crater, Mars as seen by the Navcam instrument onboard the Mars Science Laboratory rover. Icarus 249(15), 129-142 (2015). doi:10.1016/j.icarus.2014.09.02

J.R. Murphy, S. Nelli, Mars Pathfinder convective vortices: frequency of occurrence. Geophys. Res. Letters 29(23) (2002). doi:10.1029/2002GL015214

J. Murphy, C.B. Leovy, J. Tillman, Observations of martian surface winds at the Viking Lander 1 site. J. Geophys. Res. 95, B9 (1990). doi:10.1029/JB095iB09o14555

L. Neakrase et al., Particle lifting processes in dust devils. Space Sci. Rev. (2016, this issue)

L.D.V. Neakrase, R. Greeley, Dust devil sediment flux on Earth and Mars: laboratory simulations. Icarus 206, 306-318 (2010). doi:10.1016/j.icarus.2009.08.028

A.M.C. Oke, D. Dunkerley, N.J. Tapper, Willy-willies in the Australian landscape: sediment transport characteristics. J. Arid Environ. 71, 216-228 (2007)

A.V. Pathare, M.R. Balme, S.M. Metzger, A. Spiga, M.C. Towner, N.O. Renno, F. Saca, Assessing the power law hypothesis for the size-frequency distribution of terrestrial and martian dust devils. Icarus 209(2), 851-853 (2010)

J.B. Pollack, D.S. Coburn, F.M. Flasar, R. Kahn, C.E. Carslton, D. Pidek, Properties and effects of dust particles suspended in the martian atmosphere. J. Geophys. Res. 84(B6), 2929-2945 (1979)

A. Powell, Theory of vortex sound. J. Acoust. Soc. Am. 36(1), 177-195 (1964)

J. Raack, D. Reiss, G.G. Ori, K. Taj-Eddine, Vertical grain size distributions in dust devils: analyses of in situ sampled from southern Morocco. EPSC Abstract, EPSC2012-427-1, 2014

S. Rafkin, D. Banfield, J. Silver, K. Nowicki, R. Dissly, A. Stanton, in An Instrument to Measure Turbulent Fluxes in the Atmosphere of Mars and Other Planets, European Planetary Science Congress 2013. EPSC Abstracts, vol. 8, London, UK (2013), EPSC2013-575, Poster presentation, http:// meetingorganizer.copernicus.org/EPSC2013/EPSC2013-575.pdf

D. Reiss, A. Spiga, G. Erkeling, The horizontal motion of dust devils on Mars derived from CRISM and CTX/HiRISE observations. Icarus 277, 8-20 (2014)

N.O. Renno, M.L. Burkett, M.O. Larkin, A simple thermodynamical theory for dust devils. J. Atmos. Sci. 55, 3244-3252 (1998)

N.O. Renno, A.A. Nash, J. Lunine, J. Murphy, Martian and terrestrial dust devils: test of a scaling theory using Pathfinder data. J. Geophys. Res. 105(E1), 1859-1865 (2000)

N.O. Renno, V.J. Abreu, J. Koch, P.H. Smith, o.K. Hartogensis, H.A.R. De Bruin, D. Burose, G.T. Delory, W.M. Farrell, C.J. Watts, J. Garatuza, M. Parker, A. Carswell, MATADOR 2002: a pilot field experiment on convective plumes and dust devils. J. Geophys. Res. 109, E07001 (2004). doi:10.1029/ 2003JE002219

T.J. Ringrose, M.C. Towner, J.C. Zarnecki, Convective vortices on Mars: a reanalysis of Viking Lander 2 meteorological data, sols 1-60. Icarus 163(1), 78-87 (2003). doi:10.1016/S0019-1035(03)00073-3

T.J. Ringrose, M. Patel, M. Towner, M. Balme, S. Metzger, J. Zarnecki, The meteorological signatures of dust devils on Mars. Planet. Space Sci. 55, 14 (2007)

J.A. Rodriguez-Manfredi et al., MEDA: an environmental and meteorological package for Mars 2020, in 45th Lunar and Planetary Science Conference, The Woodlands, Texas, USA (2014). Poster presentation, http://ssed.gsfc.nasa.gov/IPM/PDF/1125.pdf

J.A. Ryan, J.J. Carroll, Dust devil wind velocities: mature state. J. Geophys. Res. 75, 531-541 (1970) 
J.A. Ryan, R.D. Lucic, Possible dust devils, vortices on Mars. J. Geophys. Res. 88(C15), 11005-11011 (1983)

D.S. Schmidt, R.A. Schmidt, J.D. Dent, Electrostatic force on saltating sand. J. Geophys. Res. 103(D8), 8997-9001 (1998)

J.T. Schofield, J.R. Barnes, D. Crisp, R.M. Haberle, S. Larsen, J.A. Magalhaes, J.R. Murphy, A. Seiff, G. Wilson, The Mars Pathfinder atmospheric structure investigation meteorology (ASI/MET) experiment. Science 278(5344), 1752-1758 (1997). doi:10.1126/science.278.5344.1752

R.L. Schwiesow, R.E. Cupp, M.J. Post, P.C. Sinclair, R.F. Abbey, Velocity structures of waterspouts and dust devils as revealed by Doppler lidar measurements. Bull. Am. Meteorol. Soc. 58, 677 (1977)

A. Seiff, J.E. Tillman, J.R. Murphy, J.T. Schofield, D. Crisp, J.R. Barnes, C. LaBaw, C. Mahoney, J.D. Mihalov, G.R. Wilson, R. Haberle, The atmosphere structure and meteorology instrument on the Mars Pathfinder lander. J. Geophys. Res. 103(E2), 4045-4056 (1997)

Y.P. Shao, Physics and Modelling of Wind Erosion, 2nd edn. (Springer, Heidelberg, 2008)

Y. Shao, M.R. Raupach, P.A. Findlater, Effect of saltation bombardment on the entrainment of dust by wind. J. Geophys. Res. 98, 12719-12726 (1993)

P.C. Sinclair, On the rotation of dust devils. Bull. Am. Meteorol. Soc. 46, 388-391 (1965)

P.C. Sinclair, A quantitative analysis of the dust devil. Ph.D. Dissertation, University of Arizona, 1966, p. 292

P. Sinclair, General characteristics of dust devils. J. Appl. Meteorol. 8, 32-45 (1969)

P. Sinclair, The lower structure of dust devils. J. Atmos. Sci. 30, 1599-1619 (1973)

P.H. Smith et al., Introduction to special section on the Phoenix Mission: landing site characterization experiments, mission overviews, and expected science. J. Geophys. Res. 113(E3) (2008)

P.H. Smith, M.T. Lemmon, Opacity of the Mars atmosphere measured by the imager for Mars Pathfinder. J. Geophys. Res. 104, 8975-8985 (1999)

P.H. Smith, M.G. Tomasko, D. Britt, D.G. Crowe, R. Reid, H.U. Keller, N. Thomas, F. Gliem, P. Rueffer, R. Sullivan, R. Greeley, J.M. Knudsen, M.B. Madsen, H.P. Gunnlaugsson, S.F. Hviid, W. Goetz, L.A. Soderblom, L. Gaddis, R. Kirk, The imager for Mars Pathfinder experiment. J. Geophys. Res. 102(E2), 4003-4025 (1997)

M.D. Smith, M.J. Wolff, M.T. Lemmon, N. Spanovich, D. Banfield, C.J. Budney, R.T. Clancy, A. Ghosh, G.A. Landis, P. Smith, B. Whitney, P.R. Christensen, S.W. Squyres, First atmospheric sciences results from the Mars Exploration Rovers mini-TES. Science 306(5702), 1750-1753 (2004). doi:10.1126/ science. 1104527

P.H. Smith, L.K. Tamppari, R.E. Arvidson, D. Bass, D. Blaney, W.V. Boynton, A. Carswell, D.C. Catling, B.C. Clark, T. Duck, E. DeJong, D. Fisher, W. Goetz, H.P. Gunnlaugsson, M.H. Hecht, V. Hipkin, J. Hoffman, S.F. Hviid, H.U. Keller, S.P. Kounaves, C.F. Lange, M.T. Lemmon, M.B. Madsen, M. Malin, W.J. Markiewicz, J. Marshall, C.P. McKay, M.T. Mellon, D.W. Ming, R.V. Morris, N. Renno, W.T. Pike, U. Staufer, C. Stoker, P. Taylor, J. Whiteway, A.P. Zent, Water at the Phoenix landing site. Science 325, 58-61 (2009)

J.T. Snow, T.M. McClelland, Dust devils at white sands missile range, New Mexico: 1. Temporal and spatial distributions. J. Geophys. Res. 95, 13,707-13,721 (1990)

G.G. Sorrells, A preliminary investigation into the relationship between long-period noise and local fluctuations in the atmospheric pressure field. Geophys. J. 26, 71-82 (1971)

G.G. Sorrells, J.A. McDonald, Z.A. Der, E. Herrin, Earth motion caused by local atmospheric pressure changes. Geophys. J. 26, 83-98 (1971)

S.W. Squyres et al., The Spirit rover's Athena science investigation at Gusev Crater, Mars. Science 305, 794-799 (2004)

K. Steakley, J. Murphy, A year of convective vortex activity at Gale Crater. Icarus 278, 180-193 (2016)

R. Sullivan, R. Greeley, M. Kraft, G. Wilson, M. Golombek, K. Herkenhoff, J. Murphy, P. Smith, Results of the imager for Mars Pathfinder windsock experiment. J. Geophys. Res. 105(E10), 24547-24562 (2000). doi:10.1029/1999JE001234

P.A. Taylor, D.C. Catling, M. Daly, C.S. Dickinson, H.P. Gunnlaugsson, A.M. Harri, C.F. Lange, Temperature, pressure, and wind instrumentation in the Phoenix meteorological package. J. Geophys. Res. 113, E00A10 (2008). doi:10.1029/2007JE003015

P.A. Taylor et al., On pressure measurement and seasonal pressure variations during the Phoenix mission. J. Geophys. Res. 115, E00E15 (2010). doi:10.1029/2009JE003422

J.E. Tillman, N.C. Johnson, P. Guttorp, D.B. Percival, The martian annual atmospheric pressure cycle: years without great dust storms. J. Geophys. Res. 98(E6), 10963-10971 (1993). doi:10.1029/93JE01084

A.D. Toigo, M.I. Richardson, S.P. Ewals, P.J. Gierasch, Numerical simulation of martian dust devils. J. Geophys. Res., Planets 108(E6) (2003)

M.C. Towner, Characteristics of large martian dust devils using Mars odyssey thermal emission imaging system visual and infrared images. J. Geophys. Res. 114, E02010 (2008) 
D.M. Tratt, M.H. Hecht, D. Catling, E.C. Samulon, P.H. Smith, In situ measurement of dust devil dynamics: toward a strategy for Mars. J. Geophys. Res. 108(E11), 5116 (2003)

D.T. Tyler, J.R. Barnes, Mesoscale modeling of the circulation in the Gale Crater region: an investigation into the complex forcing of convective boundary layer depths. Mars 8, 58-77 (2013). doi:10.1555/mars. 2013.0003

D.T. Tyler, J.R. Barnes, Convergent crater circulations on Mars: influence on the surface pressure cycle and the depth of the convective boundary layer. Geophys. Res. Lett. 42, 7343-7350 (2015)

J. Whiteway, M. Daly, A. Carswell, T. Duck, C. Dickinson, L. Komguem, C. Cook, Lidar on the Phoenix mission to Mars. J. Geophys. Res. 113, E00A08 (2008). doi:10.1029/2007JE003002

N.R. Williams, Development of dust whirls and similar small-scale vortices. Bull. Am. Meteorol. Soc. 29, 106-117 (1948)

J.P. Williams, Acoustic environment of the martian atmosphere. J. Geophys. Res. 106(E3), 5033-5041 (2001)

C.L. Wilson, A. Davis, D. Hutchins, M.C. Towner, An ultrasonic anemometer for Mars. J. Acoust. Soc. Am. 123(5), 3401 (2008). doi:10.1121/1.2934100

R.E. Wyett, Pressure drop in a dust devil. Mon. Weather Rev., 7-8 January 1954

L.M. Zelenyi, O.I. Korablev, D.S. Rodionov, B.S. Novikov, K.I. Marchenkov, O.N. Andreev, E.V. Larionov, Scientific objectives of the scientific equipment of the landing platform of the ExoMars-2018 mission. Sol. Syst. Res. 49(7), 509-517 (2015)

M-P. Zorzano, F.J. Martín-Torres, H. Kahanpää, J. Moores, S. Navarro, A. Lepinette, E. Sebastian, J. GómezElvira, REMS Team, The MSL Science Team), Radiation obscuration by dust devils at Gale as observed by the REMS UV sensor, in EGU General Assembly 2013. Geophysical Research Abstracts, vol. 15 (2013), EGU2013-11155, http://meetingorganizer.copernicus.org/EGU2013/EGU2013-11155.pdf 